\title{
Small amplitude oscillations before the L-H transition in EAST
}

\author{
L.M. Shao ${ }^{1}$, G.S. Xu ${ }^{1,2}$, R. Chen ${ }^{1}$, L. Chen ${ }^{1,2}$, G. Birkenmeier ${ }^{3,4}$, \\ Y.M. Duan ${ }^{1}$, W. Gao ${ }^{1}$, P. Manz ${ }^{3,4}$, T.H. Shi ${ }^{1}$, H.Q. Wang ${ }^{1}$, L. \\ Wang $^{1}$, M. Xu ${ }^{5}$, N. Yan ${ }^{1}$, L. Zhang ${ }^{1}$ and the EAST Team \\ ${ }^{1}$ Institute of Plasma Physics, Chinese Academy of Sciences, Hefei 230031, People's \\ Republic of China \\ 2 University of Science and Technology of China, Hefei 230031, People's Republic of China \\ 3 Max-Planck-Institut für Plasmaphysik, Boltzmannstr. 2, 85748 Garching, Germany \\ 4 Physik-Department E28, Technische Universität München, 85748 Garching, Germany \\ 5 Southwestern Institute of Physics, PO Box 432, Chengdu 610041, People's Republic of \\ China \\ E-mail: shaolm@ipp.ac.cn
}

October 2016

\begin{abstract}
Before L- to H-mode transition small amplitude oscillations (SAOs), different from the widely known intermediate phase (I-phase), at frequency of a few kilohertz can be observed on EAST. Under sufficient auxiliary heating SAOs can transit to H-mode or I-phase. Edge radial electric field $\left(E_{\mathrm{r}}\right)$ inside the separatrix are observed to deepen after bursts of SAOs. In SAOs the turbulence level preceding the negative radial electric field and floating potential perturbation about $90^{\circ}$ in phase, consistent with the model of zonalflows and turbulence interaction, is measured by Langmuir probe at the bottom of edge $E_{\mathrm{r}}$ well. A physical mechanism for SAOs is developed: at a critical gradient in pressure and $E_{\mathrm{r}}$, turbulence increases at the inboard edge of the $E_{\mathrm{r}}$ well. The increased turbulence level enhances the radial particle, energy and momentum transport at the plasma edge and increases the amplitude of the zonal flow at the bottom of the $E_{\mathrm{r}}$ well due to the increased Reynolds force. The increase in the zonal flow amplitude acts to mitigate the turbulence on the inboard edge of the $E_{\mathrm{r}}$ well, driving a limit-cycle oscillation. The poloidal magnetic perturbations of the oscillations are in-out/up-down asymmetric and toroidal symmetric in the SAOs.
\end{abstract}

\section{Introduction}

During the transition from low confinement mode (L-mode) to high confinement mode (Hmode), a phase with dithering cycles observed by D-alpha emission or divertor shunt current may appear [1, 2, 3, 4, 5, 6, 7]. Sanae-I Itoh et. al. first introduced this oscillating phase named 'intermediate phase' (I-phase [1]) at JFT-2M [8]. The I-phase emerges in a low density 
L-mode plasma after raising either the heating power or the density causing the turbulence to rise. A sharp transition is found when the plasma enters the I-phase [1, 9].

Before the L-H transition small amplitude oscillations (SAOs) at frequencies of a few kilohertz before the D-alpha signals or divertor shunt current dropped down, with smaller fluctuation amplitude compared to I-phase, were recently reported in several tokamaks [10, 11, 6, 12. These oscillations before a sharp L-H transition were mostly observed at EAST named 'Small-amplitude LCOs' [10, 7] and at JFT-2M termed 'Limitcycle oscillation' [11. No confinement regime transition was observed when plasmas entered the SAOs from the normal L-modes in these magnetic devices. A predator-prey model (zonal flow and turbulence competition) was proposed to explain the quasi-periodic oscillations in the SAOs and the mechanism of the L-H transition [10, 13, 5, 14, 15, 16. However, at JFT$2 \mathrm{M}$ the zonal flows were found to be too weak to trigger the oscillations of SAOs [11, 17]. Observations from many discharges at EAST confirm that the SAOs to H-mode is a sharp transition with a well-defined threshold.

The SAOs at the frequency of $1-5 \mathrm{kHz}$ are observed in normal L-modes or before $\mathrm{L}-\mathrm{H}$ transitions in EAST. These oscillations located at the plasma edge can be measured with the D-alpha filterscopes, Absolute eXtreme Ultra Violet (AXUV) diodes, Langmuir probe array at the targets and poloidal magnetic pick-up coil array. In some cases, the SAOs continue through the whole discharge under marginal power injection. The plasma states that exhibit the SAOs are different from those previously reported for the I-phase. They have a bearing on the L-H transition physics. It is therefore of high interest to characterize these SAOs and the underlying plasma state in contrast with those of the I-phase.

The paper is organized as follows. Section 2 describes the key diagnostics used in this paper, such as Absolute eXtreme Ultra Violet (AXUV) diagnostic and fast reciprocating probe. The characterization to the SAOs based on AXUV and reciprocating probe measurements is illustrated in section 3 . The differences between SAOs and I-phase in the evolution of the edge plasma quantities are described in section 4 . A physics picture of SAOs is developed and shown in section 5. The discussion and conclusions are shown in the last section.

\section{AXUV and reciprocating probe diagnostics}

To characterize the SAOs at EAST, two key diagnostics are used. One is the AXUV photodiodes [18] and the other one is the fast reciprocating probe [19]. A brief introduction to the two diagnostics is presented below.

A multi-channel photodiodes system applied for studying fast dynamics of broadband plasma radiation on the microsecond time scale was installed on EAST [18. Linear arrays of silicon photon diodes encapsulated by pinhole cameras are utilized for absolute power measurement in the AXUV spectral range. The lines of sight of the pinhole cameras each with 16 channels cross the whole poloidal cross-section of the plasma as shown in figure 1 (a). The spatial and temporal resolutions of the diagnostic are $\sim 4.5 \mathrm{~cm}$ and $10 \mu \mathrm{s}$, respectively. 

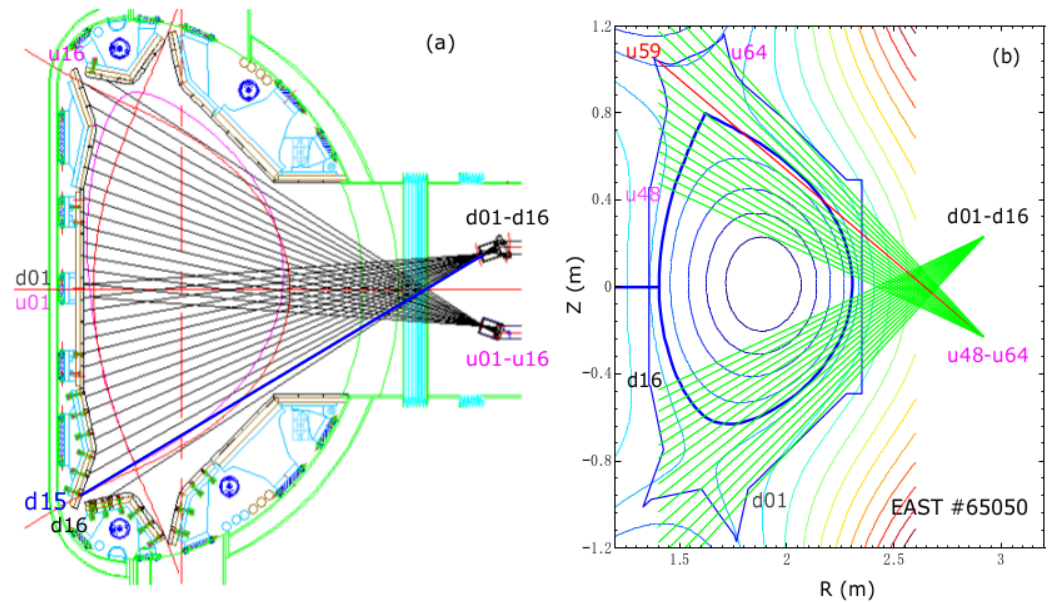

Figure 1: Lines of sight of AXUV diagnostic in the poloidal cross-section of EAST. (a) Two AXUV cameras located at outer above and below midplane in 2009. (b) Lines of sight across the upper and lower divertor since 2014. The blue and red lines of sight across the plasma edge in (a) and (b) are channels d15 and u59, respectively.

In 2014 the AXUV diagnostic was upgraded and its lines of sight across the upper and lower divertor with the spatial and temporal resolutions of $3-4 \mathrm{~cm}$ and $10 \mu \mathrm{s}$ are illustrated in figure 1(b). Photons of the vacuum-ultraviolet (VUV) spectral region penetrate the diodes and create electron-hole pairs (n-on-p junction) in the extremely thin passivation layer of the diodes. The spectral sensitivity covers a wide range of photon energies from the visible part of the spectrum up to the soft X-ray. The sensitive area of each photodiode channel is $2 \times 5 \mathrm{~mm}^{2}$ with a spacing of $0.12 \mathrm{~mm}$ between two channels.

The fast silicon AXUV diode system measures the plasma radiation between $1 \mathrm{eV}$ and $6 \mathrm{keV}$ [18]. The radiation of the plasma in the scrape-off layer is emitted mainly in the VUV region due to the low electron temperature in this region. In the lower (upper) divertor region, the radiation is mainly emitted with photon energies between 5 and $80 \mathrm{eV}$. Inside of the last closed flux surface (LCFS) the electron temperatures are higher than $80 \mathrm{eV}$, shifting the dominant photon emission to the soft X-ray region $(\sim 100-3000 \mathrm{eV})$. With the sampling rate up to $100 \mathrm{kHz}$ the AXUV diagnostic enlarges the insights into radiation dynamics of fast plasma phenomena like minor and major disruptions, edge localized modes. In this paper, however, channels just across the plasma edge, like d15 (blue line of sight) in (a) and u59 (red line of sight) in (b), are mostly used to identify the SAOs.

Dual fast reciprocating probe system was installed on EAST on the outer midplane at ports A and E, respectively [19]. The two reciprocating probes toroidally separated by $89^{\circ}$ driven by an $\mathrm{AC}$ servo motor capable of scanning a radial distance of $50 \mathrm{~cm}$ at a speed of $2 \mathrm{~m} / \mathrm{s}$. To minimize the perturbation to the edge plasma when measuring the SAOs, two small probe heads with a diameter of $17 \mathrm{~mm}$ and three tips with diamond-coated shell were utilized in the experiment. The diagram of the three-pins Langmuir probe head is shown in figure 2. The cylindrical graphite tips have a diameter of $4.5 \mathrm{~mm}$ for tips 2 and 3 as well as 


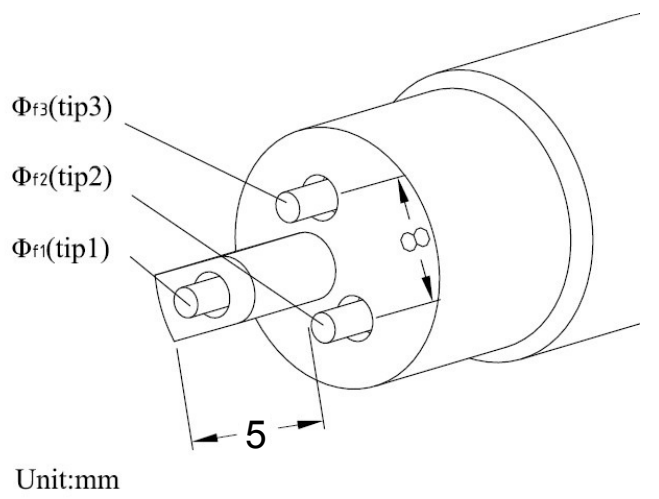

Figure 2: The diagram of the diamond-coated three-pins probe head. The cylindrical graphite tips 2 and 3 have a diameter of $4.5 \mathrm{~mm}$ and a length of $2 \mathrm{~mm}$. Tip 1 have a diameter of $1.5 \mathrm{~mm}$ and a length of $2 \mathrm{~mm}$.

$1.5 \mathrm{~mm}$ for tip 1 , and all have a length of $2 \mathrm{~mm}$. Floating potentials are measured by means of the probe with tips 2 and $3\left(\phi_{\mathrm{f} 2}\right.$ and $\left.\phi_{\mathrm{f} 3}\right)$ poloidally separated by $8 \mathrm{~mm}$ and a third tip 1 $\left(\phi_{\mathrm{f} 1}\right)$ in the middle of them. Tip 1 sticks out $5 \mathrm{~mm}$ of tips 2 and 3 in the radial direction. The sampling rate of the diagnostic is up to $5 \mathrm{MHz}$ with the data in a 12-bit digital resolution.

\section{SAOs in EAST}

The phase of SAOs before the L-H transition was investigated at EAST from 2010 [10, 7, 20]. EAST is a medium-size superconducting tokamak run since 2006 with major and minor radii ( $R$ and $a$ ) of 1.88 and $0.44 \mathrm{~m}$, respectively. The characterisation to the SAOs observed before $\mathrm{L}-\mathrm{H}$ transitions or in normal L-modes is presented below.

\subsection{Phenomenology of SAOs}

In 2010 campaign the SAOs were always observed before an L-H transition in low density plasmas heated by $2.45 \mathrm{GHz}$ lower hybrid wave (LHW). Figures 3(a)-(c) show the L-SAO$\mathrm{H}$ transition in a typical low density discharge \#36369. The plasma is run with current $I_{\mathrm{p}}=600 \mathrm{kA}$, core line-averaged density $\bar{n}_{\mathrm{e}} \sim 2.6 \times 10^{19} \mathrm{~m}^{-3}$, toroidal field $B_{\mathrm{T}}=1.76 \mathrm{~T}$ (count-clockwise direction), safety factor $q_{95}=3.25$ and biased double-null configuration (the main active X-point is located in the lower divertor). The plasma is heated by LHW with source power of $\sim 1.0 \mathrm{MW}$ as well as a slow decline of Ohmic power from 0.67 to $0.39 \mathrm{MW}$. The normal L-mode to SAOs transition indicated by the black dashed line and SAO-H transition indicated by the red dashed line happen at 3.310 and $3.348 \mathrm{~s}$, respectively. The oscillations of SAOs are well resolved by D-alpha signals measured in the lower inner and outer divertor shown in panel (a). The increased frequency of the oscillations of SAOs when the plasma accesses the SAO-H transition is often observed as illustrated by AXUV measurement of 

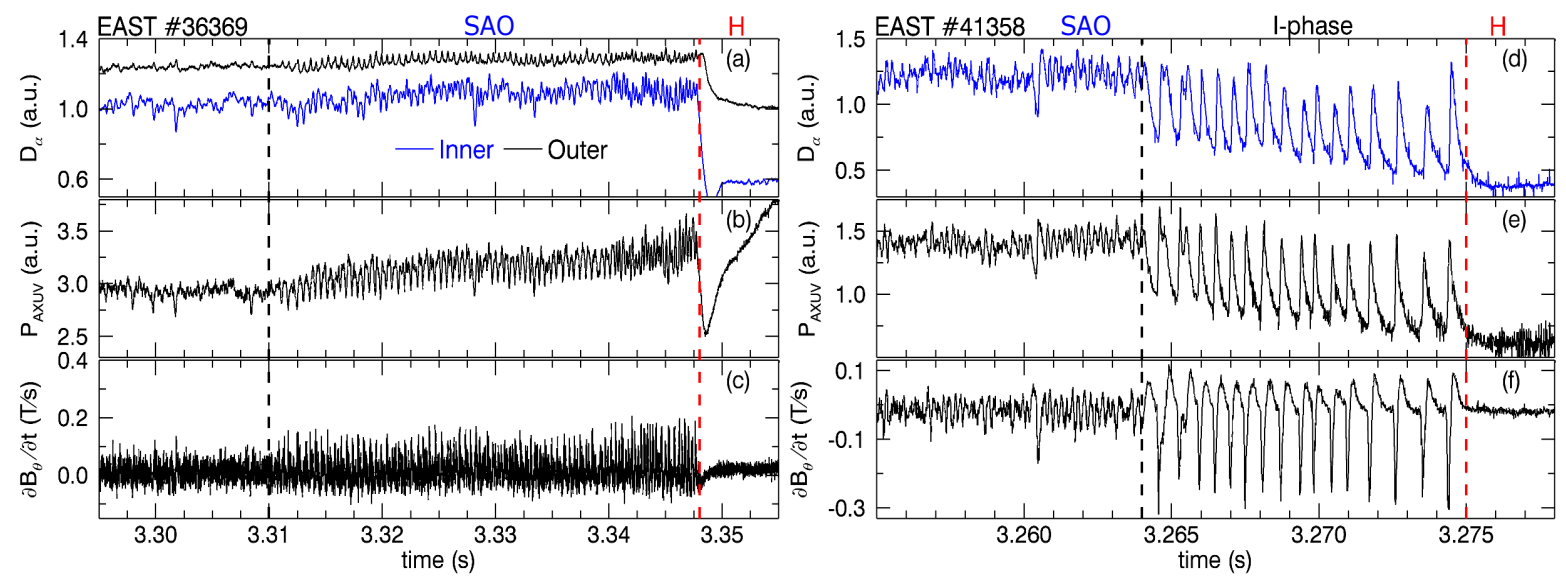

Figure 3: Temporal evolution of (a) D-alpha signals in the lower inner and outer divertor, (b) AXUV signal of channel d15 and (c) poloidal magnetic measurement of poloidal pick-up coil KMP19T located below the lower inner target of \#36369. Time trace of (d) D-alpha signal in the lower divertor, (e) AXUV signal of channel d15 and (f) magnetic measurement from pick-up coil KMP7T above the upper outer target of \#41358.

channel d15 across the plasma edge (see figure 1(a)) in panel (b). The magnetic perturbation of the oscillations of SAOs measured by poloidal magnetic pick-up coil of channel KMP19T (see figure 11(d)) located below the lower inner target is shown in panel (c). The L-SAO transition is very smooth, while both D-alpha and AXUV signals drop sharply at the SAO$\mathrm{H}$ transition. The AXUV signal recovers immediately after the transition from SAOs to H-mode.

In 2012 campaign the SAO-I-H transition, sometimes, was observed in low density plasmas as shown in figures 3(d)-(f). Discharge \#41358 is run with $I_{\mathrm{p}}=400 \mathrm{kA}$, $\bar{n}_{\mathrm{e}} \sim 2.0 \times 10^{19} \mathrm{~m}^{-3}, B_{\mathrm{T}}=1.81 \mathrm{~T}, q_{95}=4.39$ and double-null configuration. The plasma is heated by LHW and ion cyclotron resonance frequency (ICRF) with source power of $\sim 1.3$ and $\sim 0.4 \mathrm{MW}$, respectively. Both SAOs and I-phase are well resolved by D-alpha signal measured in the lower divertor, AXUV signal from channel d15 across the plasma edge and poloidal magnetic pick-up coil measurement of channel KMP7T (see figure 11(d)) located above the upper outer target. SAO-I and I-H transitions respectively happening at 3.264 and $3.275 \mathrm{~s}$ are indicated by the black and red dashed lines. When the plasma transits to the I-phase both D-alpha and AXUV signals sharply drop with an increased plasma density (not shown). This shows that SAO-I transition is a sharp transition with a increased particle confinement. The fluctuation amplitude is smaller and the frequency is higher in the oscillations of SAOs compared to I-phase illustrated in panels (d)-(f). AXUV signal acts as a good indicator for the emergence of SAOs in EAST.

Since 2013 most diagnostics at EAST are upgraded, which enable us to look more insight into the pulses of SAOs. Figure 4 shows a representative L-mode discharge \#65050 heated by $4.6 \mathrm{GHz} \mathrm{LHW}$ with a phase of SAOs. The basic parameters of the plasma is $I_{\mathrm{p}}=400 \mathrm{kA}$, 


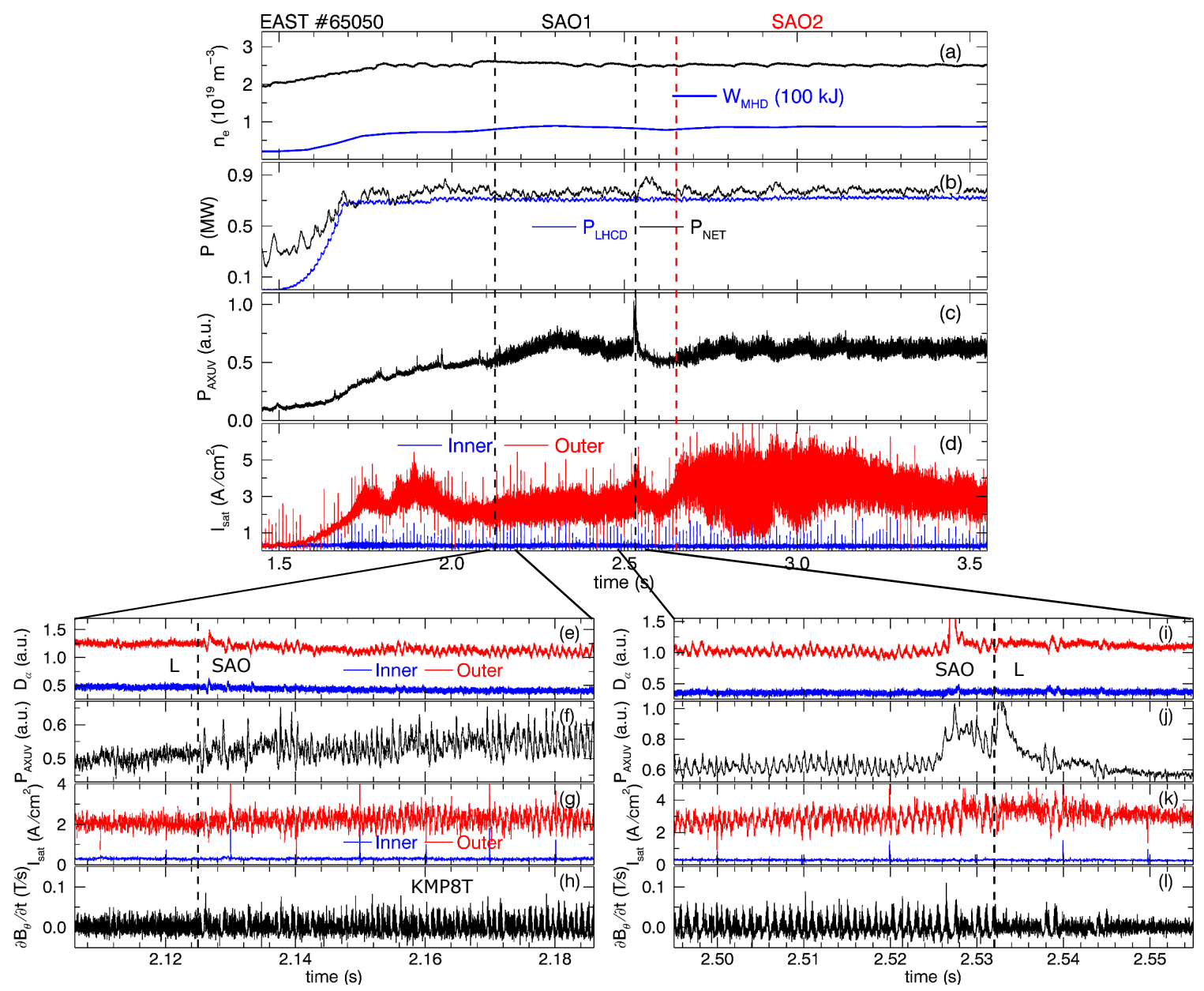

Figure 4: The time trace of (a) core line-averaged density and plasma stored energy, (b) pure injected power of lower hybrid wave and total net input power, (c) AXUV signal of channel u59 across the plasma edge and (d) ion saturated currents at upper inner and outer targets measured by Langmuir probes of \#65050. Panels (e)-(h) and (i)-(l) show two short segment of panels (c) and (d) at the beginning and termination of SAO1 phase. Panels (e) and (i) are the D-alpha signals in the upper inner and outer divertor and panels (h) and (l) show the poloidal magnetic pick-up coil measurement located above the upper outer target.

$\bar{n}_{\mathrm{e}} \sim 2.5 \times 10^{19} \mathrm{~m}^{-3}, B_{\mathrm{T}}=2.48 \mathrm{~T}$ and $q_{95}=6.45$. The magnetic configuration of this discharge is shown in figure 1 (b) and the ion $\nabla B$ drift towards the active $\mathrm{X}$-point. The time trace of the core line-averaged density and plasma stored energy $W_{\text {MHD }}$ are shown in panel (a). Panel (b) shows the injected power of 4.6 GHz LHW (subtracted by the reflecting and unabsorbed parts) and the total net input power $P_{\mathrm{NET}}$. The total net injected power values are obtained from the loss power,

$$
P_{\mathrm{NET}}=P_{\text {heat }}-d W / d t .
$$

Here, $P_{\text {heat }}$ is the sum of Ohmic and LHW heating powers subtracted by the loss and unabsorbed parts of injected power, and $W$ is the plasma stored energy. The AXUV signal 
of channel u59 (see figure 1(b)) across the plasma edge is shown in panel (c). Panel (d) shows the ion saturated currents at a sampling rate of $50 \mathrm{kHz}$ measured by the Langmuir probes at the upper inner and outer targets. When the plasma enters the SAOs phase (SAO1 2.125-2.532 s and SAO2 since $2.650 \mathrm{~s}$ ) both the core line-averaged density and plasma stored energy remain almost the same. In the meanwhile, the fluctuation amplitudes of AXUV signal of channel u59 and ion saturated current evaluated at the upper outer target increase as shown in panels (c) and (d).

Panels (e)-(h) show a short segment of panels (c) and (d) at the beginning of SAO1 phase in the time slice of 2.106-2.186 s. The L-SAO transition happens at $2.125 \mathrm{~s}$ indicated by the black dashed line. When the plasma enters the SAO1 phase the regular pulses are well resolved by the D-alpha signal at a sampling rate of $100 \mathrm{kHz}$ measured in the upper outer divertor, AXUV signal of channel u59, ion saturated current evaluated at the upper outer target and poloidal magnetic measurement of channel KMP8T (see figure 11(d)) at a sampling rate of $200 \mathrm{kHz}$. The plasma with regular oscillations return to normal L-mode at $2.532 \mathrm{~s}$ indicated by the black dashed line is shown in panels (i)-(l). The regular oscillations at the frequency of $1.1 \mathrm{kHz}$ disappear after the SAO-L transition. The dithering cycles of SAOs are not observed by D-alpha signal in the upper inner divertor and ion saturated current measured by Langmuir probe at the upper inner target in this discharge. The pulses of SAOs shown by AXUV signal are fairly coherent with the magnetic perturbation measurement. The largest fluctuation amplitude of poloidal magnetic measurements in the SAOs is at channel KMP8T. The root mean square of the poloidal magnetic perturbation $\left|\delta B_{\theta}\right|$ in the oscillations of SAOs is $\sim 0.1$ Gauss.

\subsection{Existence of $S A O s$}

Although the SAOs are observed independent of plasma current, toroidal field, ion $\nabla B$ drift direction as well as heating methods at EAST, if there is a density-dependence operational window on SAOs is unclear. Figure 5 shows an existence plot of SAOs in terms of net heating power $P_{\text {NET }}$ versus core line-averaged density in the lower hybrid wave heated plasmas with current $I_{\mathrm{p}}=600 \mathrm{kA}$ at different toroidal field. The appearance of the SAO phase has been well revealed in section 3.1. The overlaid dashed lines are the predicted $\mathrm{L}-\mathrm{H}$ transition power threshold $P_{\text {thr }, 08}$ according to the latest ITPA threshold scaling [21] choosing $B_{\mathrm{T}}=1.76 \mathrm{~T}$ (black curve), $1.88 \mathrm{~T}$ (red curve), $2.00 \mathrm{~T}$ (blue curve). Mostly, the SAOs in this dataset are observed a few milliseconds after a sawtooth crash. Please note here, the L-H transition power threshold in EAST is far above the ITPA threshold scaling [20]. Due to the constrain of the operational density in L-mode plasmas at EAST, there are no enough dataset with plasma density below 2 or above $3.5 \times 10^{19} \mathrm{~m}^{-3}$ in L-modes. SAOs, therefore, are observed throughout the accessible range of L-mode densities (not in H-mode) under sufficient heating. 


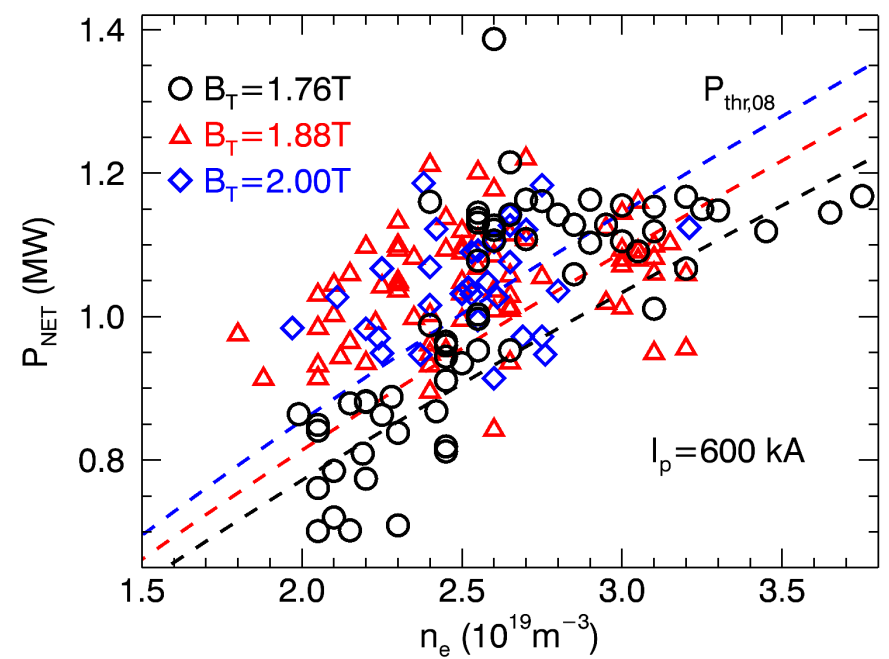

Figure 5: The existence of SAOs in terms of net input power versus core line-averaged density. The plasmas in the dataset were heated by lower hybrid wave with current of $600 \mathrm{kA}$.

\section{3. $E_{\mathrm{r}}$ and turbulence in $S A O s$}

For better characterisation to the SAOs, compensatory measurement of the edge radial electric field and turbulence level in the oscillations of SAOs by means of reciprocating probe is necessary. The edge radial electric field regularly modified by the osccillations of SAOs has been reported in reference [10]. Since the SAOs can be observed in normal L-modes and just before L-H transitions in EAST, we would like to discuss the temporal ordering of radial electric field/floating potential $\left(E_{\mathrm{r}} / \phi_{\mathrm{f}}\right)$ and turbulence in oscillations of SAOs in two cases, i.e., (1) just before L-H transition and (2) far before L-H transition in this section.

3.3.1. Just before $\mathrm{L}-\mathrm{H}$ transition Figure 6 show the L-SAO-H transition in discharge \#36369. The plasma is run with $I_{\mathrm{p}}=600 \mathrm{kA}, \bar{n}_{\mathrm{e}} \sim 2.6 \times 10^{19} \mathrm{~m}^{-3}, B_{\mathrm{T}}=1.76 \mathrm{~T}, q_{95}=3.25$ and a biased double-null configuration $\left(d R_{\mathrm{sep}}=-1 \mathrm{~cm}\right.$, the main active X-point is located in the lower divertor). The plasma is heated by LHW with source power of $\sim 1.0 \mathrm{MW}$ as shown in panel (a). The normal L-mode to SAOs transition indicated by the blue dashed line and SAO-H transition indicated by the red dashed line happen at 4.195 and 4.2635 s, respectively. In panel (b) the soft X-ray measurement across the plasma core, which reveals the crash time of sawtooth, is shown. The oscillations of SAOs are well resolved by D-alpha signals measured in the lower inner and outer divertor shown in panel (c). The increased frequency of SAOs as the plasma accessing the $\mathrm{L}-\mathrm{H}$ transition is observed by AXUV measurement of channel d15 across the plasma edge (see figure 1(a)) shown in panel (d). The spectrogram of this AXUV signal is shown in panel (e). The SAOs emerge $\sim 2 \mathrm{~ms}$ after the first sawtooth crash at the frequency of $2 \mathrm{kHz}$ and its frequency increases to $3 \mathrm{kHz}$ after the second sawtooth crash, while up to $4 \mathrm{kHz}$ when the plasma accesses to the H-mode. Both D-alpha and AXUV signals drop sharply at the SAO-H transition $\sim 9$ ms after the third sawtooth crash (4.2545 s). 


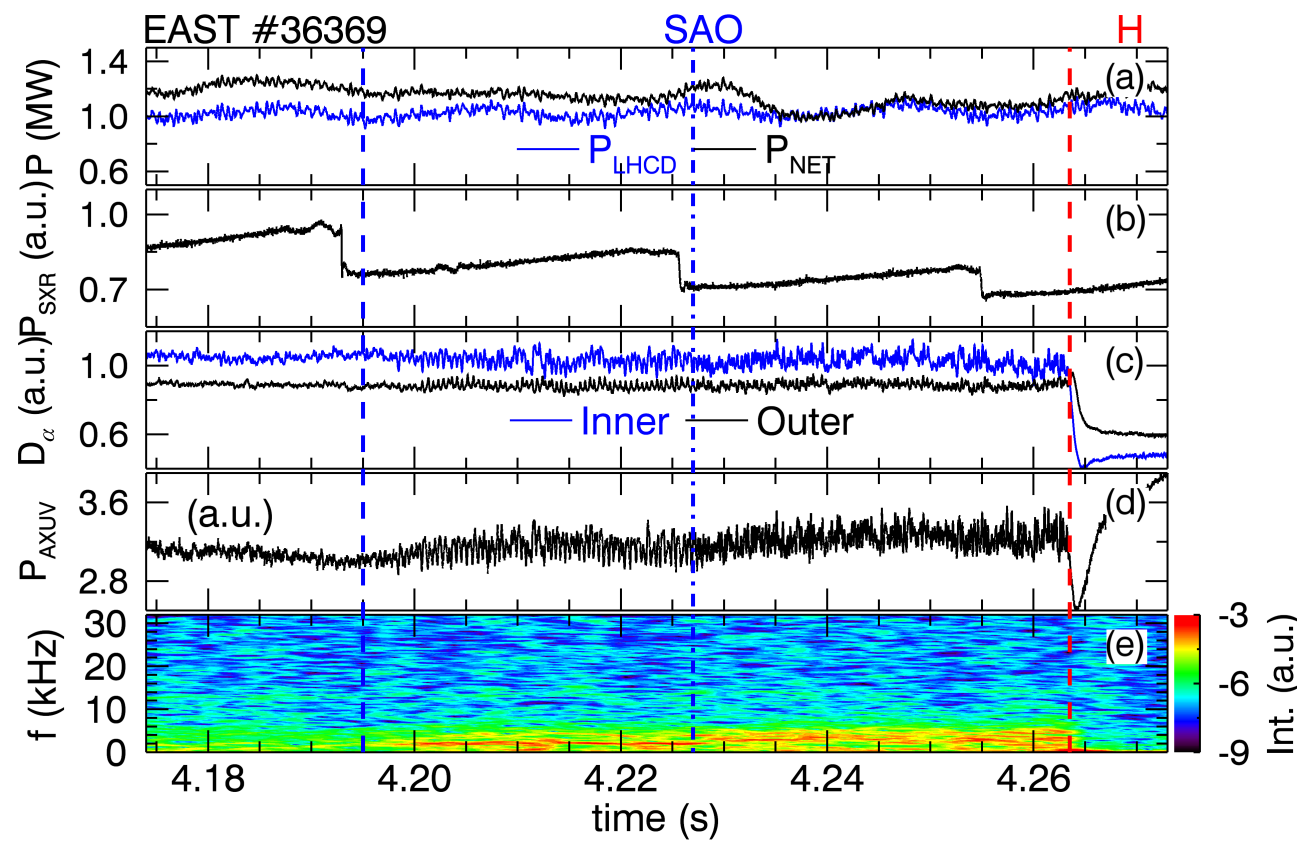

Figure 6: Time dependence of (a) lower hybrid wave power and total net input power, (b) soft X-ray measurement across plasma core, (c) D-alpha signals from inner and outer divertor, (d) AXUV signal of channel d15 and (e) its spectrogram of \#36369.

The radial electric field and turbulence measured by means of the Langmuir probe (see figure 22) at the plasma edge in the time slice of 4.260-4.265 s during the SAO-H transition of \#36369 is shown in figure 7 . The negative radial electric field $-E_{\mathrm{r}}$ (gray curve) at $r-a=-5.5 \mathrm{~mm}$ measured at port $\mathrm{E}$ is shown in panel (b). Since the probe at port A was not shot inside the separatrix, we do not show the data from the probe here. $E_{\mathrm{r}}$ shown here is evaluated as the radial floating potential gradient instead of plasma potential gradient due to lack of the electron temperature measurement, $E_{\mathrm{r}}=\left[\phi_{\mathrm{f} 1}-\left(\phi_{\mathrm{f} 2}+\phi_{\mathrm{f} 3}\right) / 2\right] / \Delta r$, i.e., no consideration of the contribution from the gradient of the electron temperature. Panel (c) shows the negative floating potential $-\phi_{\mathrm{f} 1}$ measured by the inner tip located at $r-a=-8 \mathrm{~mm}$. The turbulence level $\Phi_{\mathrm{f} 1}^{\text {env. }}$, the enveloped Hilbert transform of $\phi_{\mathrm{f} 1}$ within a band-pass filter from 25 to $250 \mathrm{kHz}$, is shown in panel (d). The turbulence level in this article is evaluated as,

$$
\begin{aligned}
& \Phi_{\mathrm{f}}^{\text {env. }}=\sqrt{\operatorname{Hilbert}^{2}\left(\tilde{\phi}_{\mathrm{f}}\right)+\left(\tilde{\phi}_{\mathrm{f}}\right)^{2}} \\
& \tilde{\phi}_{\mathrm{f}}=\phi_{\mathrm{f}}^{25-250 \mathrm{kHz}} .
\end{aligned}
$$

Here, the band-pass filter within $25-250 \mathrm{kHz}$ is applied to the input signal. The sum of the negative floating potential measured at the outer tips $\left(-\phi_{\mathrm{f} 2+\mathrm{f} 3}\right)$ is illustrated in panel (e) at $r-a=-3 \mathrm{~mm}$. In panel (f) the turbulence level $\Phi_{\mathrm{f} 2+\mathrm{f} 3}^{\text {env. }}$ at the outer tips is shown. The black, red and blue curves in panels (b)-(f) are the low-pass filtered $(<10 \mathrm{kHz})$ edge negative radial electric field, negative floating potential and turbulence level. After the SAO-H transition the edge turbulence level drops as shown in panels (d) and (f). 


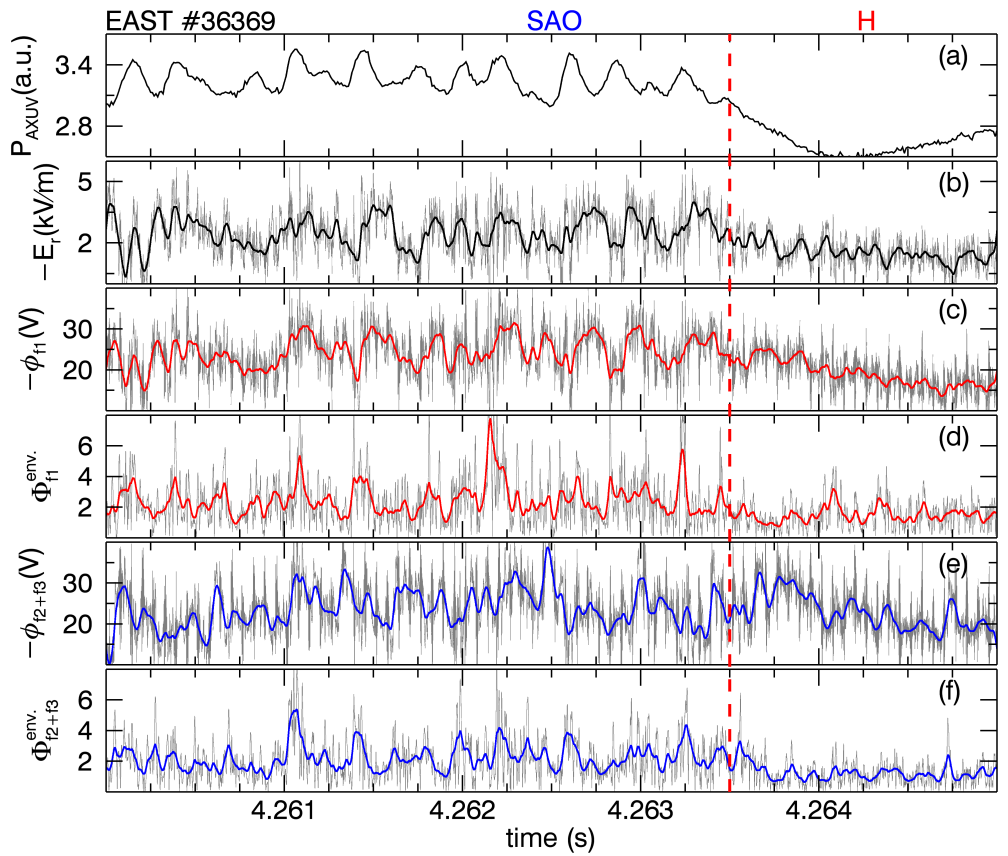

Figure 7: Time dependence of (a) AXUV signal of channel d15, (b) negative radial electric field located at $r-a=-5.5 \mathrm{~mm}$, negative floating potential at (c) $r-a=-8 \mathrm{~mm}$ and (e) $r-a=-3 \mathrm{~mm}$, and turbulence level at (d) $r-a=-8 \mathrm{~mm}$ and (f) $r-a=-3 \mathrm{~mm}$ of \#36369.

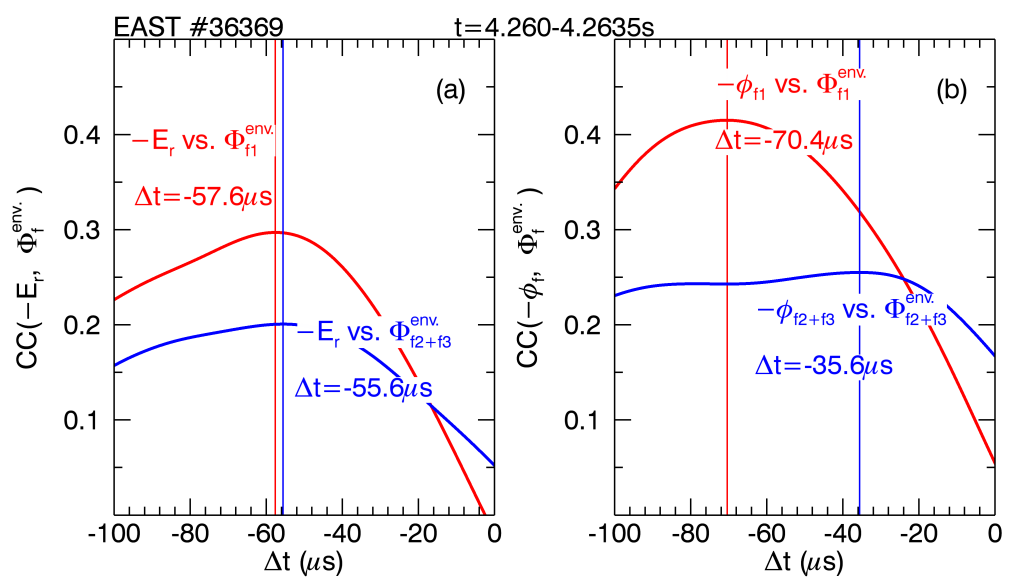

Figure 8: Cross correlations of $(\mathrm{a})-E_{\mathrm{r}}$ with edge turbulence level $\Phi_{\mathrm{f} 1}^{\text {env }}$ (inner tip, red solid curve) and $\Phi_{\mathrm{f} 2+\mathrm{f} 3}^{\text {env. }}$ (outer tips, blue solid curve) and (b) negative floating potential $-\phi_{\mathrm{f} 1}$ and $-\phi_{\mathrm{f} 2+\mathrm{f} 3}$ with turbulence level $\Phi_{\mathrm{f} 1}^{\text {env. }}$ and $\Phi_{\mathrm{f} 2+\mathrm{f} 3}^{\text {env. }}$ during the SAOs $(\sim 4 \mathrm{kHz})$ in the time slice of 4.260-4.2635 s of \#36369.

The cross correlation of the low-pass filtered $-E_{\mathrm{r}}$ with turbulence level $\Phi_{\mathrm{f} 1}^{\text {env. }}$ at the inner and outer tips is shown in Figure $8(\mathrm{a})$. The correlation of the reference signal $-E_{\mathrm{r}}$ with the signal of turbulence level is evaluated as the normalized cross-correlation function [9]. In the 
time slice of 4.260-4.2635 s the inner tip is located at the bottom of $E_{\mathrm{r}}$ well, while the outer tips locate at the outboard $E_{\mathrm{r}}$ well reconstructed by the floating potential measurement of the inner tip. The turbulence level precedes $-E_{\mathrm{r}} 57.6 \mu \mathrm{s}$ at the inner tip and $55.6 \mu \mathrm{s}$ at the outer tips, i.e., the turbulence level is ahead of the $-E_{\mathrm{r}}$ oscillations about $90^{\circ}$ in phase both at the bottom and outer board of $E_{\mathrm{r}}$ well. The turbulence level preceding the negative floating potential several decades of $\mu \mathrm{s}$ is found at the inner and outer tips (bottom and outer board of $E_{\mathrm{r}}$ well) shown in Figure 8(b). The turbulence level preceding the negative floating potential $70.4 \mu$ s, i.e., about $90^{\circ}$ in phase at the bottom of $E_{\mathrm{r}}$ well (inner tip). The temporal analysis above at the bottom and outer board of $E_{\mathrm{r}}$ well just before the SAO-H transition is well consistent with the model of zonal-flows and turbulence interaction [22] and compatible with the results of SAOs.

3.3.2. Far before $L-H$ transition Figure 9 shows the time dependence of some important edge plasma quantities in a short segment of SAOs-phase $(4.380-4.390 \mathrm{~s}, \sim 130 \mathrm{~ms}$ before the $\mathrm{L}-\mathrm{H}$ transition) of discharge \#36373 with a biased double null configuration $\left(d R_{\mathrm{sep}}=-1 \mathrm{~cm}\right)$. The plasma is heated by LHW with source power of $\sim 1.0 \mathrm{MW}$ in addition to $\sim 0.4 \mathrm{MW}$ Ohmic power at $\bar{n}_{\mathrm{e}} \sim 3.0 \times 10^{19} \mathrm{~m}^{-3}, I_{\mathrm{p}}=0.6 \mathrm{MA}, B_{\mathrm{T}}=1.76 \mathrm{~T}$ and $q_{95}=3.34$. The oscillations of SAOs at a frequency of $\sim 2 \mathrm{kHz}$ are resolved by AXUV signal of channel d15 (see figure 1(a)) across the plasma edge shown in panel (a). In the time slice of figure 9 tip 1 of the three-pins probe was inserted into the edge plasma at $r-a \sim-10 \mathrm{~mm}$ and, thus, tips 2 and 3 were located at $r-a \sim-5 \mathrm{~mm}$. $-E_{\mathrm{r}}$ (gray curve) located at $r-a \sim-7.5 \mathrm{~mm}$ is shown in panel (b). The edge turbulence level at tip 1 is shown in panel (c). In panel (d) the envelope signal of $\phi_{\mathrm{f} 2}+\phi_{\mathrm{f} 3}$ is shown. The smoothed color curves in panels (b)-(d) are the low-pass filtered $(<10 \mathrm{kHz})-E_{\mathrm{r}}$ and edge turbulence level, which are more convenient to compare

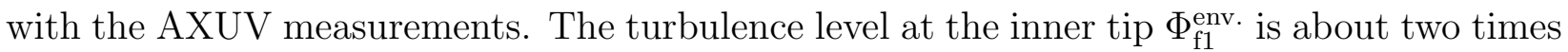
higher than that at the outer tips $\Phi_{\mathrm{f} 2+\mathrm{f} 3}^{\text {env }} / 2$ as shown in panels (c) and (d). The oscillations of SAOs illustrated by the AXUV signal and $-E_{\mathrm{r}}$ are fairly coherent, and are consistent to the evolution of the edge turbulence level.

The delay-time between the low-pass filtered edge turbulence level $\Phi_{\mathrm{f} 1}^{\text {env. }}$ and $\Phi_{\mathrm{f} 2+\mathrm{f} 3}^{\text {env. }}$ with $-E_{\mathrm{r}}$ is shown in panel (e). The edge turbulence level $\Phi_{\mathrm{f} 1}^{\text {env. }}$ lags $-E_{\mathrm{r}}$ oscillations $-3.2 \mu \mathrm{s}$ (red solid line), and $\Phi_{\mathrm{f} 2+\mathrm{f} 3}^{\text {env }}$ lags $-E_{\mathrm{r}}$ oscillations $2.2 \mu \mathrm{s}$ (blue dashed line) in the time slice of 4.380-4.390 s. This means that $-E_{\mathrm{r}}$ oscillations at $r-a \sim-7.5 \mathrm{~mm}$ could be in phase with turbulence level at $r-a \sim-10$ and $-5 \mathrm{~mm}$ if taking the radial propagation time of the turbulence from inner to outer tips into account. We map the radial positions of the tips to the edge $E_{\mathrm{r}}$ well reconstructed by the floating potential measurement of the inner tip and find: the inner and outer tips respectively locating at the inboard and bottom of $E_{\mathrm{r}}$ well.

As discussed above we can not clearly tell the temporal ordering of $-E_{\mathrm{r}}$ and turbulence level in the oscillations of SAOs at the tips of the reciprocating probe far before the L$\mathrm{H}$ transition. However, the temporal ordering of the floating potential perturbation and turbulence level is possible with one probe pin as shown in figure 10. Panels (a)-(e) show a short segment of the SAOs phase in \#36373 (see figure 9) in the time slice of 4.3875-4.390 s. 

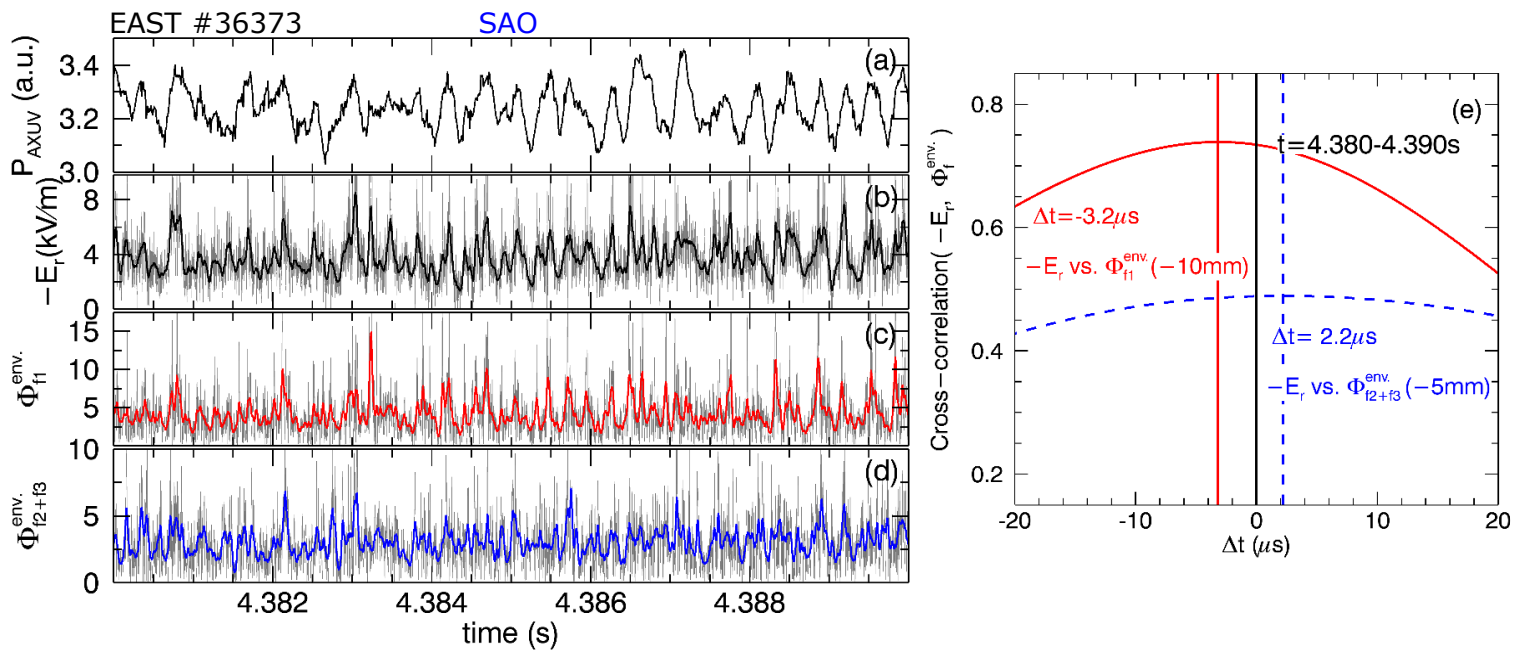

Figure 9: Temporal evolution of (a) AXUV signal of channel d15, (b) negatively radial electric field located at $r-a \sim-7.5 \mathrm{~mm}$ and edge turbulence level located at $r-a \sim-10 \mathrm{~mm}$ (c) and $-5 \mathrm{~mm}(\mathrm{~d})$ in a short segment of SAOs phase of discharge \#36373. The red curves in panels (b)-(d) are the low-pass filtered $-E_{\mathrm{r}}$ and edge turbulence level. Panel (e) shows the cross correlations of $-E_{\mathrm{r}}$ with edge turbulence level $\Phi_{\mathrm{f} 1}^{\text {env. }}$ (inner tip) and $\Phi_{\mathrm{f} 2+\mathrm{f} 3}^{\text {env. }}$ (outer tips) in the time slice of $4.380-4.390 \mathrm{~s}$.
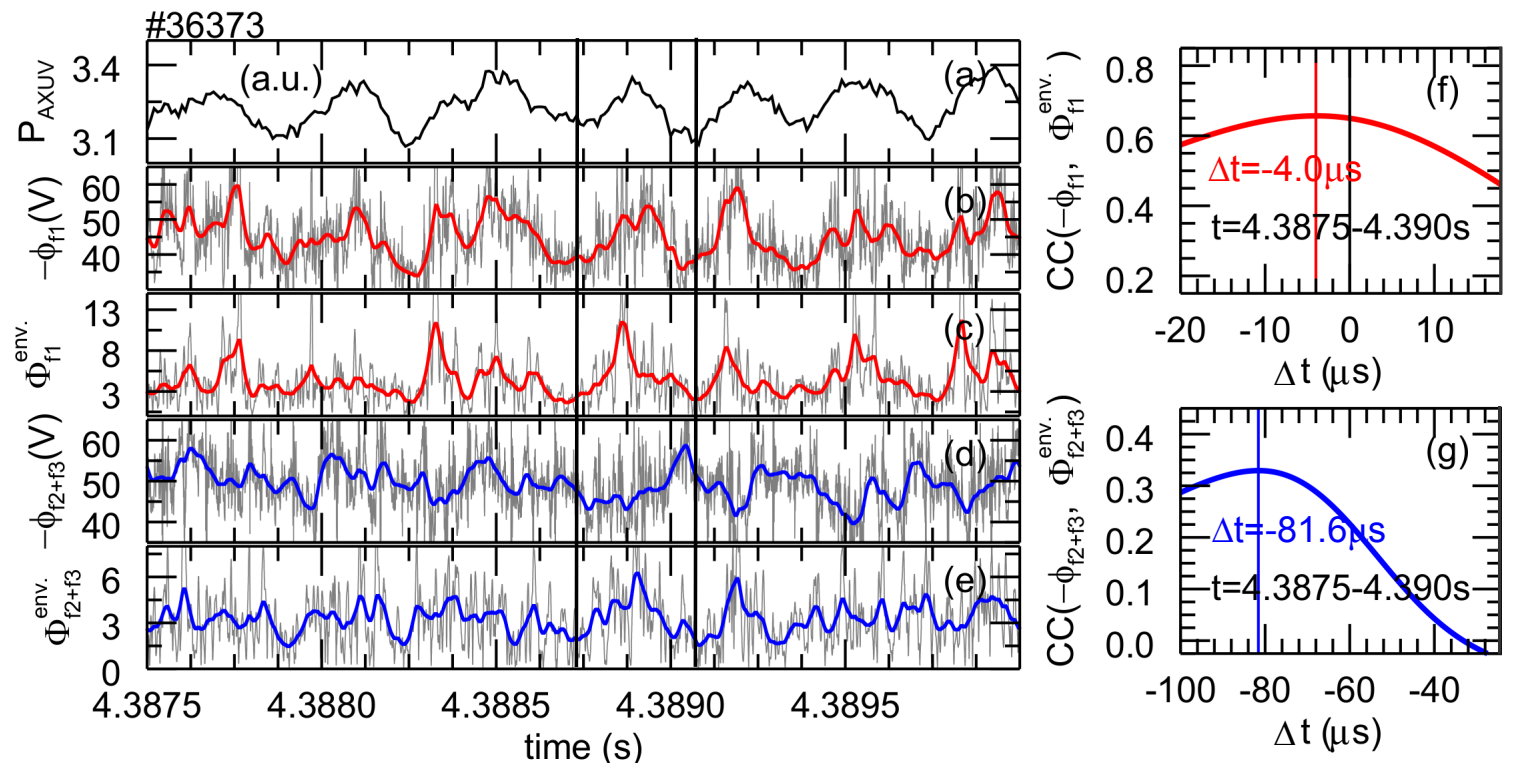

Figure 10: Temporal evolution of (a) AXUV signal of channel d15, (b) negative floating potential fluctuation and (c) turbulence level at tip 1, (d) sum of negative floating potential fluctuation and (e) turbulence level at tips 2 and 3 of a short segment of the SAOs in \#36373. Cross correlations of the negative floating potential fluctuation with edge turbulence level at the (f) inner tip and (g) outer tips. 
The negative floating potential fluctuation $-\phi_{\mathrm{f} 1}$ (gray curve) measured by tip 1 is shown in panel (b). The sum of the negative floating potential fluctuation measured at tips 2 and 3 $\left(-\phi_{\mathrm{f} 2+\mathrm{f3}}\right)$ is illustrated in panel (d). The red and blue curves in panels (b)-(e) are the lowpass filtered $(<10 \mathrm{kHz})$ floating potential fluctuation and edge turbulence level. Panels (f) and $(\mathrm{g})$ show the cross correlations of the low-pass filtered floating potential fluctuation with edge turbulence level measured at inner tip (red curve) and outer tips (blue curve). In this time slice the edge turbulence level precedes the negative floating potential perturbation $4.0 \mu \mathrm{s}$ at the inner tip and $81.6 \mu \mathrm{s}$ at the outer tips. The burst of turbulence in front of the increase of floating potential perturbation at the outer tips is well shown in panels (d) and (e) indicated by two vertical black lines. In the time slice of 3.3875-3.390 s there are 7 oscillations, therefore, each pulse has a $357.1 \mu$ s time length. $\frac{81.6 \mu \mathrm{s}}{357.1 \mu \mathrm{s}} \sim \frac{1}{4}$, i.e., the phase of the turbulence level precedes the negative floating potential perturbation about $90^{\circ}$ at the outer tips (bottom of $E_{\mathrm{r}}$ well) while only $\sim 4^{\circ}$ is found at the inner tips (inboard $E_{\mathrm{r}}$ well). The analysis at the outer tip is consistent with the model of zonal-flows and turbulence interaction [22], since in first order to zonal flow is a potential perturbation with a finite radial wave number. In the time slice of figure 10 the low-pass filtered floating potential perturbation at the inner tip precedes $50.0 \mu \mathrm{s}$ than that at the outer tips. The radial phase velocity of the low frequency perturbation of the floating potential is estimated as $\frac{5 \times 10^{-3} \mathrm{~m}}{50.0 \times 10^{-6} \mathrm{~s}}=100 \mathrm{~m} / \mathrm{s}$.

\subsection{Magnetic structure of SAOs}

As mentioned in section 3.1, the oscillations of SAOs can be detected by the poloidal magnetic pick-up coils. Figure 11 shows a short segment of SAOs phase with magnetic perturbation measurements in the time slice of 2.250-2.258 s (see figure 4) of \#65050. The oscillations of SAOs resolved by AXUV signal of channel u59 (see figure 1(b)) are shown in panel (a). Two poloidal arrays of magnetic pick-up coils at port $\mathrm{K}$ and port $\mathrm{C}$ (KMPT and CMPT) each with 24 channels covering the whole poloidal circumference toroidally separated by $180^{\circ}$ are illustrated in panels (d). The poloidal magnetic fluctuation measured by channels KMP20T, KMP19T, KMP17T, KMP1T, KMP2T, KMP4T, KMP6T, KMP8T, KMP11T KMP14T, KMP26T, KMP24T, KMP21T normalised to their root-mean-square values and applied a low-pass filter is shown in panel (b). Panel (c) shows the normalised magnetic perturbation measured by channels CMP20T, CMP19T, CMP17T, CMP1T, CMP2T, CMP4T, CMP6T, CMP8T, CMP11T, CMP13T, CMP26T, CMP24T, CMP21T applied a low pass filter at port $\mathrm{C}$. The poloidal magnetic perturbation is larger in the divertor region than that in the mid-plane in the SAOs. The pick-up coil measurements are updown asymmetric (KMP20T/KMP19T versus KMP6T/KMP8T, CMP20T/CMP19T versus CMP6T/CMP8T) in the SAOs as shown in panels (b) and (c), which is consistent to the AXUV measurements of channel u59 and d05 (not shown here). In the meanwhile, a very nice in-out asymmetry in SAOs is also observed, see KMP1T/KMP2T versus KMP14T/KMP26T and CMP1T/CMP2T versus CMP13T/CMP26T. This in-out asymmetry can be a sign of zonal-flow activity [23, 24]. 

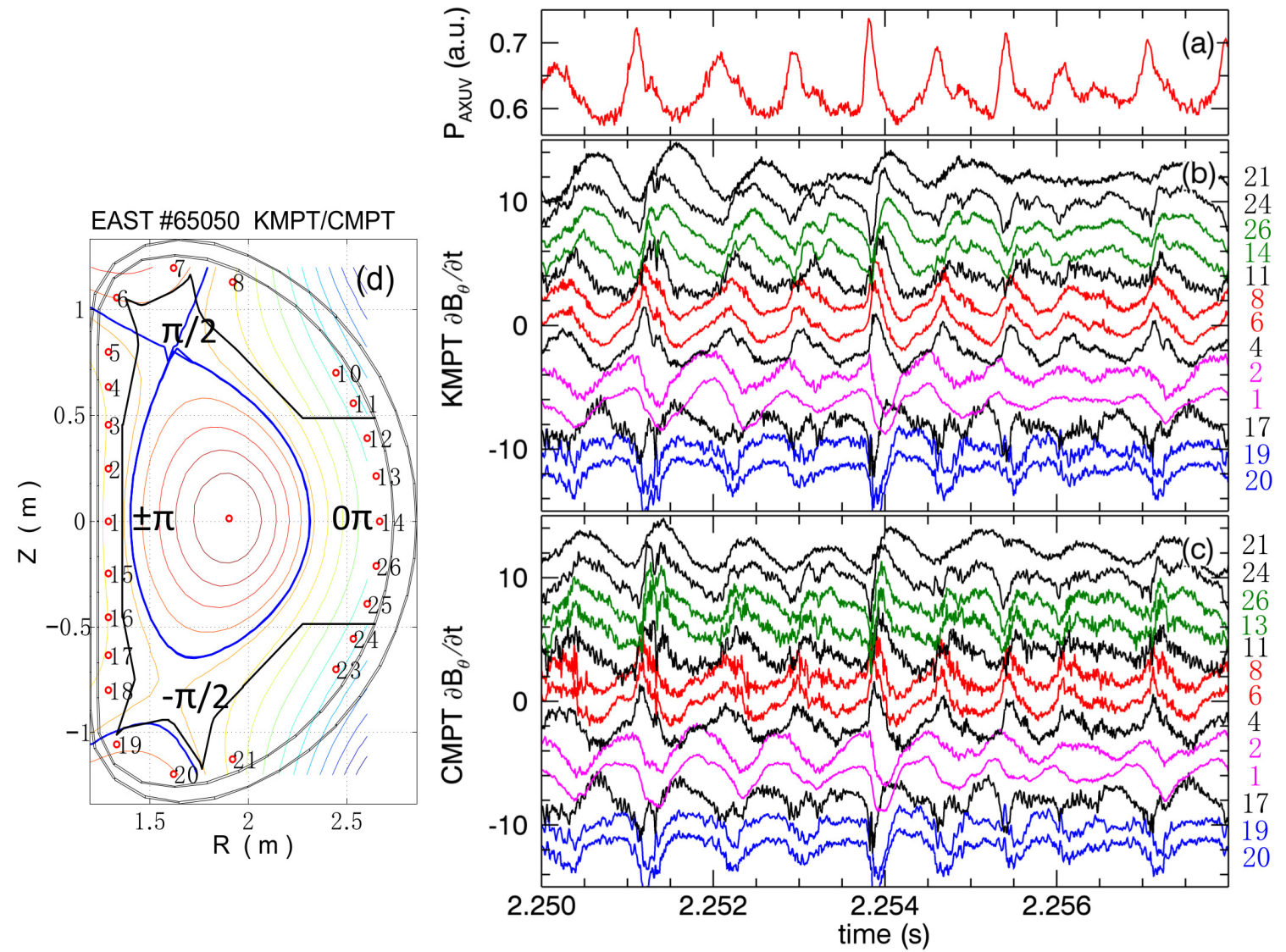

Figure 11: Time dependence of (a) AXUV signal from channel u59 across the plasma edge, poloidal magnetic pick-up coil measurements from (b) poloidal array KMPT and (c) poloidal array CMPT applied a low pass filter in a short segment of SAOs-phase of \#65050. The distribution of the poloidal arrays of magnetic pick-up coils (d) at port $\mathrm{K}$ and port $\mathrm{C}$ toroidally separates by $180^{\circ}$.

Comparing panels (b) and (c) we find that the magnetic perturbations between the two arrays are nearly in phase for all poloidal positions, suggesting that the magnetic perturbations of SAOs are axisymmetric in the toroidal direction. The hypothesis is confirmed by the measurement of a 16 channels poloidal magnetic pick-up coil array uniformly distributed in the toroidal circumference at the low field side. This proposes a toroidal mode number of $n=0$ magnetic structure of SAOs at EAST.

The propagation of the magnetic perturbation of SAOs is in the clockwise direction in the poloidal cross-section (ion-diamagnetic direction at the high field side) as shown in figure 12 . Here, we choose the magnetic probe KMP14T as a reference and cross-correlate its normalised signal with all other pick-up coil signals in the time slice of $2.245-2.285 \mathrm{~s}$. The reference magnetic probe is located at the outer midplane with poloidal angle $\theta \approx 0$ (see figure $11(\mathrm{~d})$ ). At the low field side $(\theta \approx 0)$ and high field side $(\theta \approx \pm \pi)$ the correlation is highest and opposite, corresponding to the in-out asymmetry of the poloidal magnetic perturbation. High 


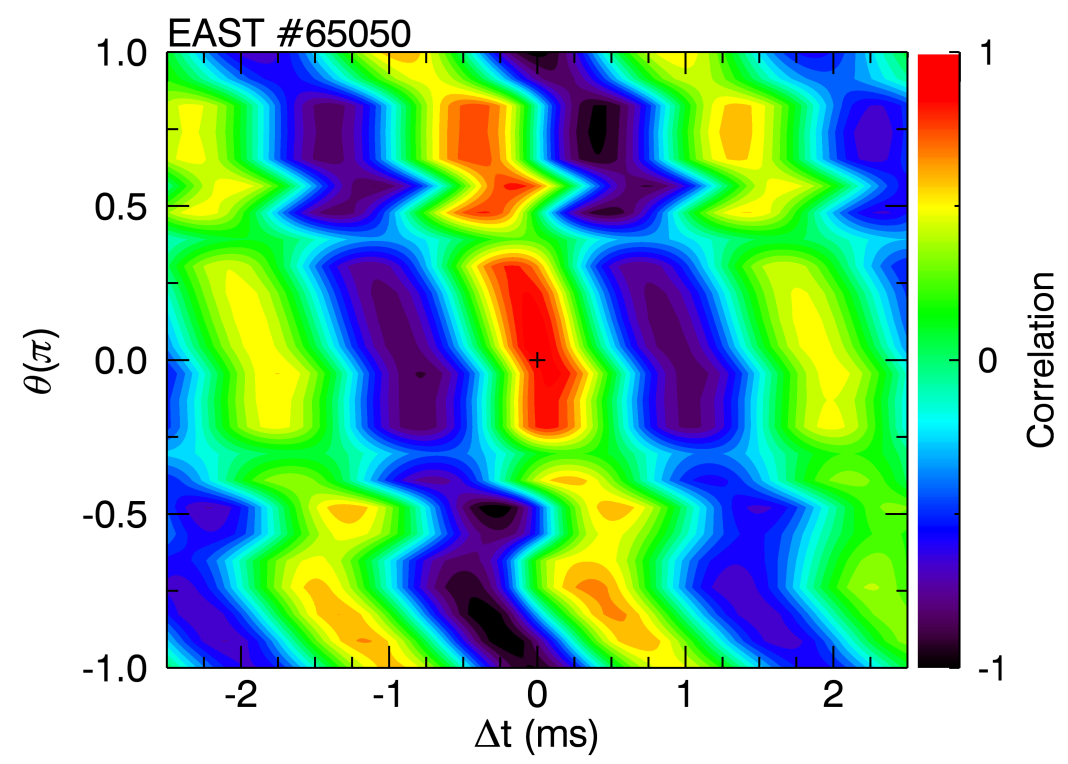

Figure 12: Poloidal mode structure of the polodial magnetic pick-up coil signals (KMPT) from cross-correlation analysis with the reference magnetic pick-up coil of KMP14T (black cross).

and opposite sign values are also found at the bottom $(\theta \approx-\pi / 2)$ and top $(\theta \approx \pi / 2)$ of the plasma, which is in consistence with the observed up-down asymmetric magnetic fluctuations. The red stripe of positive correlation reveals a propagation from the outer midplane to the bottom and back to the outer midplane along the poloidal circumference in the clockwise direction. Phase analysis shows that the oscillations of SAOs have a poloidal mode number of $\mathrm{m}=1$ magnetic structure.

\section{Differences between SAOs and I-phase}

The SAOs to I-phase transition is a sharp confinement regime transition with a less particle transport as discussed in section 3.1. Although some different phenomena between them were shown in figures $3(\mathrm{~d})-(\mathrm{f})$, a more detailed comparison of the plasma parameters between them at the plasma edge is necessary.

Edge plasma parameters during SAO-I transition can be given by a reciprocating probe array in the outer midplane. Figure 13 shows the layout of the $3 \times 4$ (poloidal $\times$ radial) probe array, which provides the floating potential, ion saturated current and electron temperature and density with a temporal resolution of $1 \mu \mathrm{s}$. The probe array is a replacement head for the fast reciprocating probe described in sections 2 . Three tips on each layer are poloidally spaced by $d p=6 \mathrm{~mm}$ and four layers of tips are spaced by $d r=2.5 \mathrm{~mm}$ in the radial direction. All tips in the probe array are $2 \mathrm{~mm}$ in length and $2 \mathrm{~mm}$ in diameter. Quantities $\phi_{\mathrm{f} 1}-\phi_{\mathrm{f} 8}$ and $\phi_{+1} / \phi_{+2}$ are the floating potential and positively biased potential, respectively. $I_{\mathrm{s} 1}$ and $I_{\mathrm{s} 2}$ are the measurements of the ion saturated current. 3 tips at the first layer and 


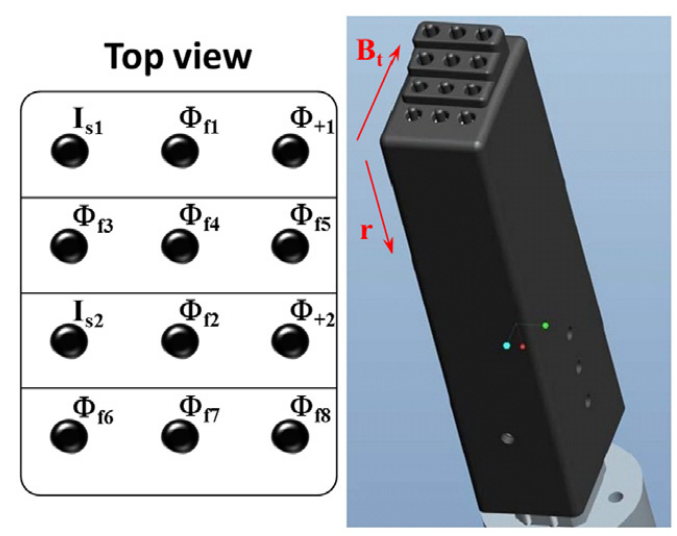

Figure 13: Layout of $3 \times 4$ probe array with three tips on each layer poloidally spaced by $d p=6 \mathrm{~mm}$ and four layers of tips radially spaced by $d r=2.5 \mathrm{~mm}$. Each tip is $2 \mathrm{~mm}$ in length and $2 \mathrm{~mm}$ in diameter.

third layer form a triple probe.

Figure 14 shows a SAO-I transition discharge \#42160 heated by LHW and ICRF at source power of $\sim 1.5$ and $\sim 0.9 \mathrm{MW}$, respectively. The plasma was run with $I_{\mathrm{p}}=0.4 \mathrm{MA}$, $\bar{n}_{\mathrm{e}} \sim 2.3 \times 10^{19} \mathrm{~m}^{-3}, B_{\mathrm{T}}=1.80 \mathrm{~T}$ and a biased double null configuration $\left(d R_{\text {sep }}=1.5 \mathrm{~cm}\right.$, the main active $\mathrm{X}$-point is located in the upper divertor). In this discharge the innermost tips $\left(I_{\mathrm{s} 1}, \phi_{\mathrm{f} 1}\right.$ and $\left.\phi_{+1}\right)$ were inserted into the edge plasma at $r-a \sim-5.0 \mathrm{~mm}$. Thus, tips $I_{\mathrm{s} 2}, \phi_{\mathrm{f} 2}$ and $\phi_{+2}$ on the third layer were located at $r-a \sim 0.0 \mathrm{~mm}$ (separatrix). Panel (a) shows the D-alpha signal measured in the lower divertor and AXUV signal of channel u15 (see figure 1(a)) across the plasma edge. Edge electron densities evaluated from the probe measurements respective at $r-a \sim-5.0\left(n_{\mathrm{e} 1}\right.$, gray curve) and $0.0 \mathrm{~mm}\left(n_{\mathrm{e} 2}\right)$ are illustrated in panel (b). Panel (c) shows the electron temperatures located at $r-a \sim-5.0$ ( $T_{\mathrm{e} 1}$, gray curve) and $0.0 \mathrm{~mm}\left(T_{\mathrm{e} 2}\right)$, respectively. The red and blue solid lines in panels (b) and (c) are the low-pass filtered edge plasma parameters. The electron temperature is evaluated as $T_{\mathrm{e}}=\left(\phi_{+}-\phi_{\mathrm{f}}\right) / \ln 2$, where $\phi_{+}$is the potential fluctuation measured by the positively biased tip. The electron density is estimated as $n_{\mathrm{e}}=I_{\mathrm{s}} /\left(0.5 e A_{\mathrm{eff}} C_{\mathrm{s}}\right)$, where $I_{\mathrm{s}}$ is the ion saturated current, $e$ is the electric charge, $A_{\text {eff }}$ is the effective collecting area of the tip and $C_{\mathrm{s}}=\sqrt{2 T_{\mathrm{i}} / m_{\mathrm{i}}}$ is the sound speed (here, using $T_{\mathrm{i}}=T_{\mathrm{e}}$ ). Electron pressure gradient evaluated as $\nabla P_{\mathrm{e}}=\left(n_{\mathrm{e} 2} T_{\mathrm{e} 2}-n_{\mathrm{e} 1} T_{\mathrm{e} 1}\right) /(2 d r)$ at $r-a \sim-2.5 \mathrm{~mm}$ is shown in panel (d). Negative poloidal velocity in the negative (ion diamagnetic drift) direction and negative poloidal flow shear respectively estimated as $-<V_{\theta}>=<\left(\phi_{\mathrm{f} 1}-\phi_{\mathrm{f} 2}\right)>/\left(2 d r B_{\mathrm{T}}\right)$ and $-\partial<V_{\theta}>/ \partial r=<\left(\phi_{\mathrm{f} 1}-\phi_{\mathrm{f} 4}\right)-\left(\phi_{\mathrm{f} 4}-\phi_{\mathrm{f} 2}\right)>/\left(d r^{2} B_{\mathrm{T}}\right)$ at $r-a \sim-2.5 \mathrm{~mm}$ are also shown in panel (d). Reynolds stress at $r-a \sim-2.5 \mathrm{~mm}$ shown in panel (e) is calculated as RS $=<\left(\tilde{\phi}_{\mathrm{f} 4}-\tilde{\phi}_{\mathrm{f} 1}\right)\left(\tilde{\phi}_{\mathrm{f} 5}-\tilde{\phi}_{\mathrm{f} 4}\right)>/\left(d p d r B_{\mathrm{T}}^{2}\right)$. Here, the floating potential perturbations are calculated from the Langmuir probe potential measurements applying a band-pass filter within $20-250 \mathrm{kHz}$.

The SAO-I transition happening at $3.9947 \mathrm{~s}$ is indicated by a black solid vertical line with 


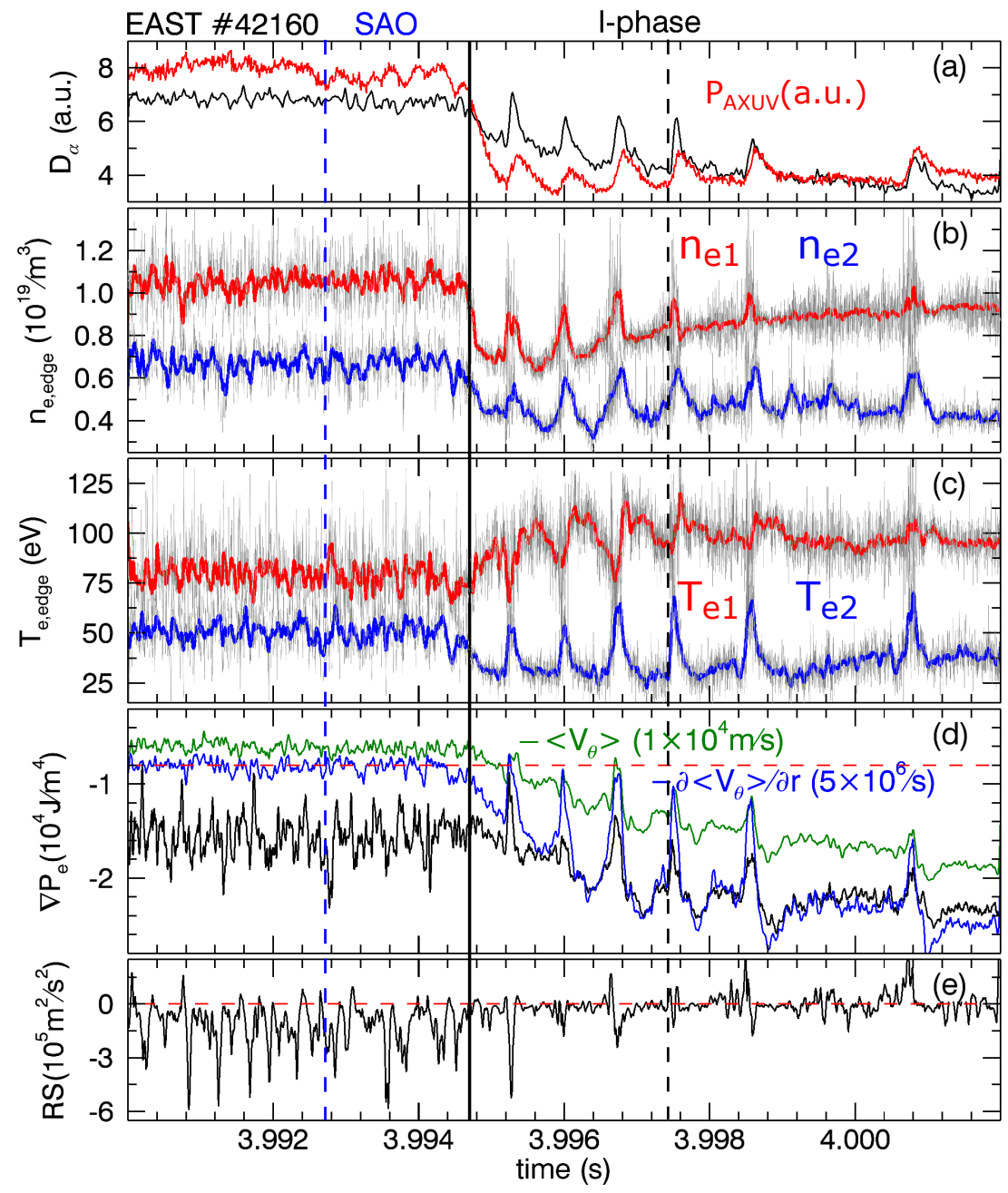

Figure 14: Time trace of (a) D-alpha signal in the lower divertor and AXUV signal of channel u15, (b) edge electron density $n_{\mathrm{e} 1}$ and $n_{\mathrm{e} 2}$ at $r-a \sim-5.0$ and $0.0 \mathrm{~mm}$, (c) electron temperature $T_{\mathrm{e} 1}$ and $T_{\mathrm{e} 2}$ at $r-a \sim-5.0$ and $0.0 \mathrm{~mm},(\mathrm{~d})$ radial diamagnetic electric field $E_{\mathrm{r}, \text { dia }}$, poloidal velocity and negative poloidal flow shear at $r-a \sim-2.5 \mathrm{~mm}$ and (e) Reynolds stress at $r-a \sim-2.5 \mathrm{~mm}$.

a sharp decrease of D-alpha and AXUV signals as well as electron densities at $r-a \sim-5.0$ and $0.0 \mathrm{~mm}$, respectively. Starting from the confinement regime transition the electron temperature at $r-a \sim-5.0 \mathrm{~mm}$ increases while decreases at the separatrix corresponding to a deepened edge $\nabla P_{\mathrm{e}} . \nabla P_{\mathrm{e}}$ deepens when an oscillation of SAOs bursts indicated by the blue dashed vertical line. The deepened $\nabla P_{\mathrm{e}}$ is in accordance of the increased $-E_{\mathrm{r}}$ after each oscillation burst of SAOs at the plasma edge shown in figure 9(b). While $\nabla P_{\mathrm{e}}$ relaxes after the disruption of each pulse of I-phase indicated by the black dashed vertical line. The Reynolds stress is mostly negative in the SAOs phase, while around zero in the I-phase. The Reynolds stress is still negative after the burst of earlier pulses of I-phase, however, the sign changes in the later pulses of I-phase. Before the L-H transition $-\partial<V_{\theta}>/ \partial r$ is stable 


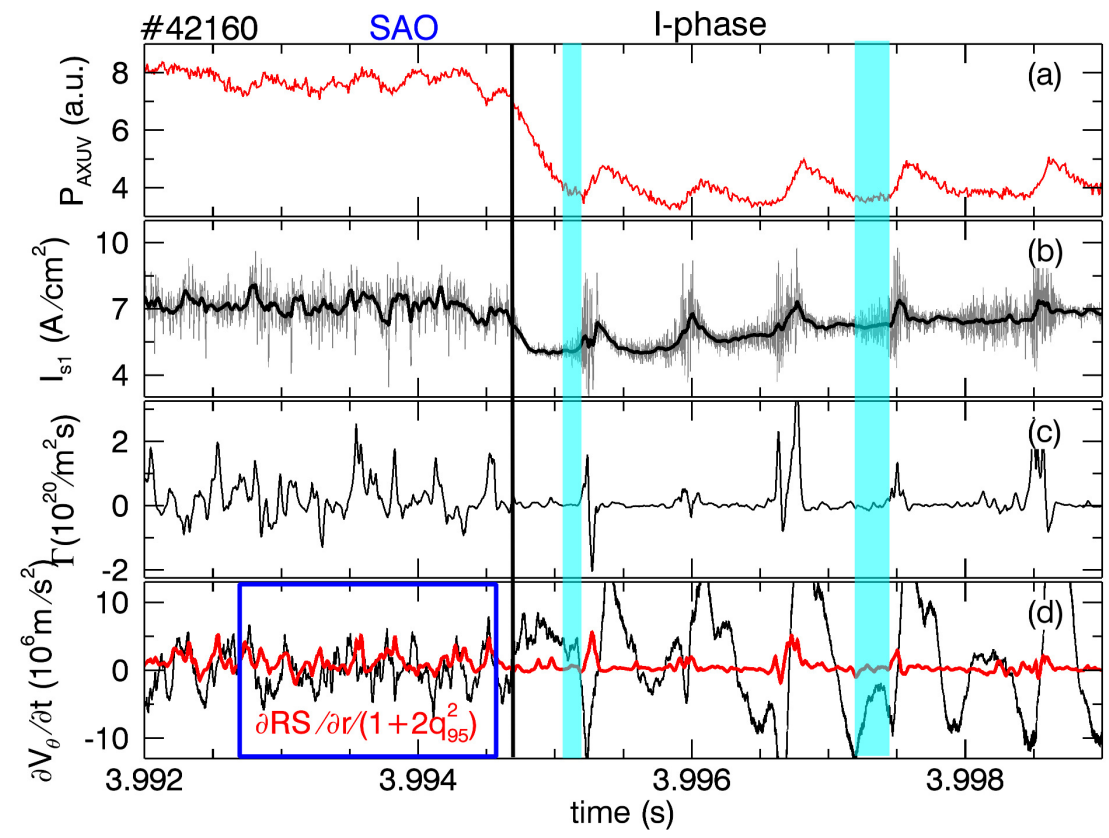

Figure 15: Time dependence of (a) AXUV signal of channel u15, (b) ion saturated current at $r-a \sim-5.0 \mathrm{~mm}$, (c) particle flux at $r-a \sim-2.5 \mathrm{~mm}$ and (d) accelerated speed of ploidal velocity and Reynolds stress gradient with a factor of $1 /\left(1+2 q_{95}^{2}\right)$ at $r-a \sim-2.5 \mathrm{~mm}$ of discharge \#42160.

at $\sim-4 \times 10^{19} \mathrm{~s}^{-1}$, while increases up to $-5 \times 10^{19} \mathrm{~s}^{-1}$ just before the SAO-I transition at 3.9944 s. Please note here, a sawtooth crashed at $3.992 \mathrm{~s}$ before the L-SAO (and SAO-I) transition. Therefore, we suppose that the heat flux from the core by auxiliary heating or released by the sawtooth crash plays a key role on SAOs and SAO-I (SAO-H) transition.

In figure 14(b) the precursor of some pulses of I-phase at $\sim 100 \mathrm{kHz}$ is observed in density fluctuation measurement at $r-a \sim-5.0 \mathrm{~mm}$. The precursor in the density fluctuation is originally from the measurement of the ion saturated current as shown in figure 15(b) (cyan regions). However, no precursor is observed before the bursts of the oscillations of SAOs in the ion saturated current measurement. The precursor of the pulses of I-phase is not resolved by the floating potential measurements (not shown). Obviously outward particle flux, $\Gamma=<\tilde{n}_{\mathrm{e} 1} \tilde{v}_{\mathrm{r} 1}>$, driven by transiently enhanced turbulence level is observed in both oscillations of SAOs and pulses of I-phase shown in figure 15(c). Here, the radial advection velocity fluctuation is estimated as $\tilde{v}_{\mathrm{r} 1}=\left(\tilde{\phi}_{\mathrm{f} 5}-\tilde{\phi}_{\mathrm{f} 4}\right) /\left(d p B_{\mathrm{T}}\right)$. The accelerated speed of ploidal velocity $\partial<V_{\theta}>/ \partial t \approx \partial<\phi_{\mathrm{f} 2}-\phi_{\mathrm{f} 1}>/ \partial t /\left(2 d r B_{\mathrm{T}}\right)$ and its one component driven by the Reynolds force $\partial<\mathrm{RS}>/ \partial \mathrm{r} /\left(1+2 \mathrm{q}_{95}^{2}\right)$ [25, 11] at $r-a \sim-2.5 \mathrm{~mm}$ are shown in panel (d). Here, the Reynolds stress gradient is estimated as $\partial<\mathrm{RS}>/ \partial \mathrm{r}=\left[<\left(\tilde{\phi}_{\mathrm{f} 2}-\tilde{\phi}_{\mathrm{f} 4}\right)\left(\tilde{\phi}_{\mathrm{f} 5}-\tilde{\phi}_{\mathrm{f} 4}\right)>-<\left(\tilde{\phi}_{\mathrm{f} 4}-\tilde{\phi}_{\mathrm{f} 1}\right)\left(\tilde{\phi}_{\mathrm{f} 5}-\tilde{\phi}_{\mathrm{f} 4}\right)>\right] /\left[d p\left(d r B_{\mathrm{T}}\right)^{2}\right]$ and $q_{95}=4.28$. The value of the accelerated speed driven by the Reynolds force (red curve) is comparable to the poloidal accelerated speed shown in the blue rectangle during the oscillation bursts of SAOs, which is different from the result in JFT-2M where the oscillatory 
Reynolds stress is too small to accelerate the SAOs flow [11, 17].

\section{Physics picture of SAOs}

Based on the analysis above, a physical mechanism for the oscillations of SAOs is proposed below. At a critical gradient in pressure and $E_{\mathrm{r}}$, turbulence increases at the inboard edge of the $E_{\mathrm{r}}$ well. The increased turbulence level enhances the radial particle, energy and momentum transport at the plasma edge and increases the amplitude of the zonal flow at the bottom of the $E_{\mathrm{r}}$ well due to the increased Reynolds force. The increase in the zonal flow amplitude acts to mitigate the turbulence on the inboard edge of the $E_{\mathrm{r}}$ well, driving a limit-cycle oscillation.

The proposed physical picture is supported by the experimental evidence that the SAOs emerged after a sawtooth crash as shown in figure 6. The oscillations of SAOs appear to be triggered by the heat pulse of sawtooth. In section $3.3 E_{\mathrm{r}}$ and turbulence level in the oscillations of SAOs are well resolved by the fast reciprocating probe at $\sim 10 \mathrm{~mm}$ (inner tip) and just a few millimeters (outer tips) inside the separatrix. Just before the SAO$\mathrm{H}$ transition, the phase of turbulence level preceding $-E_{\mathrm{r}}$ about $90^{\circ}$ in phase is observed at the bottom and outer board of $E_{\mathrm{r}}$ well. The turbulence level preceding the negative floating potential perturbation about $90^{\circ}$ is also found at the bottom of $E_{\mathrm{r}}$ well; For case of SAOs far before the L-H transition, only a few microseconds of delay time between $-E_{\mathrm{r}}$ and turbulence level are found in the $E_{\mathrm{r}}$ well. Nevertheless, the turbulence level precedes the negative floating potential perturbation about $90^{\circ}$ measured at the bottom of $E_{\mathrm{r}}$ well. This is still in accordance with the model of zonal-flows and turbulence interaction [22], since in first order to zonal flow is a potential perturbation. Similar results were found in TJ-K stellerator measured by Langmuir probe array [26]. Poloidal momentum analysis in figure 15 shows that the oscillatory Reynolds stress is not small to accelerate the oscillating flow of SAOs. Recently, critical edge $E_{\mathrm{r}}$ are found to play key roles on the L-H transition physics and power threshold [27, 12] and SAOs have been reported before the L-H transition [12] at ASDEX Upgrade. While in JET, the stationary zonal-flows in the edge $E_{\mathrm{r}}$ well in Ohmic and L-mode discharges [28] are resolved by a high spatial resolution Doppler backscattering.

The magnetic pick-up coil measurements show that the oscillations of SAOs have a magnetic $\mathrm{m}=1 / \mathrm{n}=0$ (poloidal/radial) structure. The magnetic structure of the oscillations in SAOs is very similar to that of I-phase reported in EAST [7, HL-2A [4] and ASDEX Upgrade [9, 23]. This magnetic structure respectively propagating in the ion/electron diamagnetic direction along the high/low field side is found in SAOs and I-phase [7] both with counter-clockwise $B_{\mathrm{T}}$ (view from the top) and clockwise $I_{\mathrm{p}}$ at EAST. Taking the particle ejection in the oscillations of SAOs and I-phase into account, we explain the magnetic $\mathrm{m}=1$ structure as following. When an oscillation of SAOs or I-phase bursts the volume of the plasma (inside the last closed flux surface) increases a bit caused by the particle ejection, i.e., the toroidal magnetic flux $\Phi_{B}=\oint B_{\mathrm{T}} r d l$ rises. Here, $B_{\mathrm{T}}$ is in the counter-clockwise direction. According to the Faraday's law of electromagnetic induction the electromotive 
force, $\varepsilon=-d \Phi_{B} / d t$, in the poloidal cross-section is produced at the plasma edge in the counter-clockwise direction. Then an electrons cluster at the very plasma edge is accelerated by $\varepsilon$ in the clockwise direction along the polodial circumference. The accelerated electrons cluster propagates starting from the outer midplane and back to the outer midplane in the clockwise direction, strongly coupled to the up-down asymmetric pressure sideband and the Pfirsch-Schlüter current [23, 29], during an oscillation of SAOs or I-phase is detected by the poloidal magnetic pick-up coil array. Thus, the poloidal magnetic mode number $\mathrm{m}=1$ in SAOs and I-phase due to the up-down asymmetric pressure sideband. At the low field side of the plasma accelerated blobs were observed in the electron diamagnetic direction in an I-phase pulse measured by the gas puff imaging diagnostic at EAST [3].

Intensive investigation of I-phase and its physical interpretation has been done already in Refs. 1, 2, 5, 7, 14, 22, 30, 31]. The pulses of I-phase are popularly explained as zonal-flows and turbulence interaction based on the predator-prey model [22, 30]. However, Langmuir probe measurements in HL-2A show that there is another limit cycles in which the pressuregradient-induced drift plays the essential role [4. X. Q. Wu, et. al. recently propose an one-dimensional model for I-phase [31] that the pressure gradient acts as the predator at the inboard of edge $E_{\mathrm{r}}$ well while the zonal-flows play the predator at the outer board of $E_{\mathrm{r}}$ well. This model still can not explain the two types of limit-cycle oscillations observed at the inner side of the edge $E \times B$ flow shear layer close to the $\mathrm{L}-\mathrm{H}$ transition threshold condition in the TJ-II stellarator [32]. At ASDEX Upgrade magnetic precursors [9] and mean flows dominating the $E \times B$ velocity [33] are reported in I-phase. The $E \times B$ velocity dominated by the mean flows is consistent with the result shown in figure 15(d) where the Reynolds force is much smaller than the acceleration of the poloidal velocity in I-phase. Therefore, the classical model for the I-Phase oscillations [22, 30] can not interpret all the experimental observations, nevertheless, is in accordance with the proposed mechanism for SAOs above.

\section{Discussion and conclusions}

Small amplitude oscillations (SAOs) at frequencies of a few kilohertz (1-5 kHz) in normal L-modes or before L-H transitions with sufficient heating are observed at EAST. These oscillations can be measured by D-alpha signals, AXUV photodiodes, divertor Langmuir probe array as well as poloidal magnetic pick-up coils. The SAOs are observed independently of plasma current, density, toroidal field, ion $\nabla B$ drft direction and heating methods. The plasma density and stored energy keeps the same (with a low gas fuelling rate) and no confinement regime transition is found in SAOs. Similar regular oscillations are also observed by gas puff imaging in NSTX [34], H-alpha or D-alpha signal in W7-AS [35] and TCV [36] and by beam emission spectroscopy [37] in DIII-D before the L-H transitions. At ASDEX Upgrade, if SAOs are observed in an L-mode plasma before the confinement regime transition then an I-phase always follows [12]. Nevertheless, I-Phase is not often observed during the $\mathrm{SAO}-\mathrm{H}$ transitions at EAST.

The SAO-I transition is a confinement regime transition with a less particle transport. 
Edge pressure gradient $\nabla P_{\mathrm{e}}$ deepens when a oscillation of SAOs bursts, while relaxes after the disruption of each pulse of I-phase. Poloidal momentum analysis shows that the oscillatory Reynolds stress seems to be strong enough to accelerate the oscillating flow of SAOs. The precursor before the pulses of I-phase is observed in the measurement of ion saturated current, while no precursor is found before the bursts of the oscillations of SAOs. The heat flux from the core by auxiliary heating or released by sawtooth crashes is supposed to play the key role on triggering SAOs and SAO-I (SAO-H) transition [38.

A physical picture to explain the oscillations of SAOs is proposed in section 5 of this article. The magnetic pick-up coil measurements show that the oscillations of SAOs are in-out/up-down asymmetric and toroidal symmetric corresponding to a magnetic $m=1 / n=0$ structure. Based on the measurements of fast reciprocating probe, we can speculate that the SAOs are generated by an interaction of zonal-flows with turbulence as already discussed in the context of the I-phase [22, 30]. Other effects like atomic physics in the divertor or unknown states of edge turbulence could be likewise responsible for the SAO dynamics. It will be a subject of the future investigations.

\section{Acknowledgments}

The authors would like to thank J. Cheng, Y-K. M. Peng, E. Wolfrum and S. J. Zweben for constructive discussions. This work was supported by National Magnetic Confinement Fusion Science Program of China under Contracts No. 2015GB101000 and National Natural Science Foundation of China under Contract No.11575235 and No.11422546.

\section{References}

[1] G. D. Conway, C. Angioni, F. Ryter, P. Sauter, J. Vicente, and ASDEX Upgrade Team, Phys. Rev. Lett. 106, 065001 (2011).

[2] L. Schmitz, L. Zeng, T. L. Rhodes, J. C. Hillesheim, E. J. Doyle, R. J. Groebner, W. A. Peebles, K. H. Burrell, and G. Wang, Phys. Rev. Lett. 108, 155002 (2012).

[3] L. M. Shao, G. S. Xu, S. C. Liu, S. J. Zweben, B. N. Wan, H. Y. Guo, A. D. Liu, R. Chen, B. Cao, W. Zhang, H. Q. Wang, L. Wang, S. Y. Ding, N. Yan, G. H. Hu, H. Xiong, L. Chen, Y. L. Liu, N. Zhao, and Y. L. Li, Plasma Phys. Controlled Fusion 55, 105006 (2013).

[4] J. Cheng, J. Q. Dong, K. Itoh, L. W. Yan, M. Xu, K. J. Zhao, W. Y. Hong, Z. H. Huang, X. Q. Ji, W. L. Zhong, D. L. Yu, S. I. Itoh, L. Nie, D. F. Kong, T. Lan, A. D. Liu, X. L. Zou, Q. W. Yang, X. T. Ding, X. R. Duan, Yong Liu, and HL-2A Team, Phys. Rev. Lett. 110, 256002 (2013).

[5] G. R. Tynan, M. Xu, P. H. Diamond, J. A. Boedo, I. Cziegler, N. Fedorczak, P. Manz, K. Miki, S. Thakur, L. Schmitz, L. Zeng, E. J. Doyle, G. M. McKee, Z. Yan, G. S. Xu, B. N. Wan, H. Q. Wang, H. Y. Guo, J. Dong, K. Zhao, J. Cheng, W. Y. Hong, and L. W. Yan, Nucl. Fusion 53, 073053 (2013).

[6] S. H. Müller, G. D. Conway, G. Birkenmeier, D. Carralero, T. Happel, A. Herrmann, P. Manz, de P. Marne, A. Mlynek, H. W. Müller, S. Potzel, V. S. Rohde, U. Stroth, M. Tsalsa, G. R. Tynan, E. Wolfrum, and ASDEX Upgrade Team, Phys. Plasmas 21, 042301 (2014).

[7] G. S. Xu, H. Q. Wang, M. Xu, B. N. Wang, H. Y. Guo, P. H. Diamond, G. R. Tynan, R. Chen, N. Yan, D. F. Kong, H. L. Zhao, A. D. Liu, T. Lan, V. Naulin, A. H. Nielsen, J. Juul Rasmussen, K. Miki, P. Manz, W. Zhang, L. Wang, L. M. Shao, S. C. Liu, L. Chen, S. Y. Ding, N. Zhao, Y. L. Li, Y. L. Liu, G. H. Hu, X. Q. Wu, and X. Z. Gong, Nucl. Fusion 54, 103002 (2014). 
[8] Sanae-I Itoh, Kimitaka Itoh, Atsushi Fukuyama, Yukitoshi Miura, and JFT-2M Group, Phys. Rev. Lett. 67, 2485 (1991).

[9] G. Birkenmeier, M. Cavedon, G. D. Conway, P. Manz, U. Stroth, R. Fischer, G. Fuchert, T. Happel, F. M. Laggner, M. Maraschek, A. Medvedeva, V. Nikolaeva, D. Prisiazhniuk, T. Pütterich, F. Ryter, L. M. Shao, M. Willensdorfer, E. Wolfrum, H. Zohm, and the ASDEX Upgrade Team, Nucl. Fusion 56, 086009 (2016).

[10] G. S. Xu, B. N. Wan, H. Y. Guo, H. L. Zhao, A. D. Liu, V. Naulin, P. H. Diamond, G. R. Tynan, M. Xu, R. Chen, M. Jiang, P. Liu, N. Yan, W. Zhang, L. Wang, S. C. Liu, and S. Y. Ding, Phys. Rev. Lett. 107, 125001 (2011).

[11] T. Kobayashi, K. Itoh, T. Ido, K. Kamiya, S. I. Itoh, Y. Miura, Y. Nagashima, A. Fujisawa, S. Inagaki, K. Ida, and K. Hoshino, Phys. Rev. Lett. 111, 035002 (2013).

[12] L. M. Shao, E. Wolfrum, G. Birkenmeier, S. Potzel, M. Bernert, M. Cavedon, G. D. Conway, R. Fischer, F. M. Laggner, P. Manz, M. Maraschek, F Mink, M. Willensdorfer, and the ASDEX Upgrade Team, IPP Report 17, 51 (2016).

[13] P. Manz, G. S. Xu, B. N. Wan, H. Q. Wang, H. Y. Guo, I. Cziegler, N. Fedorczak, C. Holland, S. H. Müller, S. C. Thakur, M. Xu, K. Miki, P. H. Diamond, and G. R. Tynan, Phys. Plasmas 19, 072311 (2012).

[14] I. Cziegler, G. R. Tynan, P. H. Diamond, A. E. Hubbard, J. W. Hughes, J. Irby, and J. L. Terry, Nucl. Fusion 55, 083007 (2015).

[15] M. A. Malkov, P. H. Diamond, K. Miki, J. E. Rice, and G. R. Tynan, Phys. Plasmas 22, 032506 (2015).

[16] G. R. Tynan, I. Cziegler, P. H. Diamond, M. Malkov, A. Hubbard, J. W. Hughes, J. L. Terry, and J. H. Irby, Plasma Phys. Control. Fusion 58, 044003 (2016).

[17] K. Itoh, T. Kobayashi, T. Ido, S-I. Itoh, and K. Kamiya, Plasma Phys. Control. Fusion 57, 092001 (2015).

[18] Yanmin DUAN, Liqun HU, Songtao MAO, Ping XU, Kaiyun CHEN, Shiyao LIN, Guoqiang ZHONG, Jizong ZHANG, Ling ZHANG, and Liang WANG, Plasma Sci. Technol. 13, 546 (2011).

[19] W. Zhang, J. F. Chang, B. N. Wan, G. S. Xu, C. J. Xiao, B. Li, G. S. Xu, N. Yan, L. Wang, S. C. Liu, M. Jiang, and P. Liu, Rev. Sci. Instrum. 81, 113501 (2010).

[20] L. Chen, G. S. Xu, A. H. Nielsen, W. Gao, Y. M. Duan, H. Q. Liu, L. Wang, M. H. Li, M. Wang, X. J. Zhang, R. Chen, H. Q. Wang, Z. Sun, S. Y. Ding, N. Yan, S. C. Liu, L. M. Shao, W. Zhang, G. H. Hu, J. Li, L. Zhang, B. N. Wan, and the EAST Team, Nucl. Fusion 56, 056013 (2016).

[21] Y. R. Martin, T. Takizuka, and ITPA CDBM H-mode Threshold Database Working Group, J. Phys.: Conf. Ser. 123, 012033 (2008).

[22] Eun-jin Kim and P. H. Diamond, Phys. Rev. Lett. 90, 185006 (2003).

[23] P. Manz, G. Birkenmeier, G. Fuchert, M. Cavedon, G. D. Conway, M. Maraschek, A. Medvedeva, F. Mink, B. D. Scott, L. M. Shao, U. Stroth, and ASDEX Upgrade Team, Phys. Plasmas 23, 052302 (2016).

[24] Bruce D Scott, New J. Phys. 7, 92 (2005).

[25] K. Itoh and S-I. Itoh, Plasma Phys. Control. Fusion 38, 1 (1996).

[26] G. Birkenmeier, M. Ramisch, B. Schmid, and U. Stroth, Phys. Rev. Lett. 110, 145004 (2013).

[27] P. Sauter, T. Pütterich, F. Ryter, E. Viezzer, E. Wolfrum, G. D. Comway, R. Fischer, B. Kurzan, R. M. McDermott, S. K. Rathgeber, and the ASDEX Upgrade Team, Nucl. Fusion 52, 012001 (2012).

[28] J. C. Hillesheim, E. Delabie, H. Meyer, C. F. Maggu, L. Meneses, E. Poli, and JET Contributors EUROfusion Consortium, JET, Culham Science Centre, Abingdon, Oxon OX14 3DB, United Kingdom, Phys. Rev. Lett. 116, 065002 (2016).

[29] Y. Camenen, A. G. Peeters, C. Angioni, F. J. Casson, W. A. Hornsby, A. P. Snodin, and D. Stribtzi, Phys. Plasmas 16, 062501 (2009).

[30] K. Miki, P. H. Diamond, Ö. D. Gürcan, G. R. Tynan, T. Estrada, L. Schmitz, and G. S. Xu, Phys. Plasmas 19, 092306 (2012).

[31] Xingquan Wu, Guosheng Xu, Baonian Wan, Jens Juul Rasmussen, Volker Naulin, and Anders Henry 
Nielsen, Nucl. Fusion 55, 053029 (2015).

[32] T. Estrada, E. Ascasíbar, E. Blanco, A. Cappa, F. Castejón, C. Hidalgo, B. Ph. Van Milligen, and title = E. Sánchez, .

[33] M. Cavedon, T. Pütterich, E. Vizzer, G. Birkenmeier, T. Happel, F. M. Laggner, P. Manz, F. Ryter, U. Stroth, and the ASDEX Upgrade Team, Nucl. Fusion 57, 014002 (2017).

[34] S. J. Zweben, R. J. Maqueda, R. Hager, K. Hallatschek, S. M. Kaye, T. Munsat, F. M. POli, A. L. Roquemore, Y. Sechrest, and D. P. Stotler, Phys. Plasmas 17, 102502 (2010).

[35] F. Wagner, Plasma Phys. Control. Fusion 49, B1 (2007).

[36] Y. R. Martin and TCV team, Plasma Phys. Control. Fusion 46, A77 (2004).

[37] Z. Yan, G. R. McKee, R. Fonck, P. Gohil, R. J. Groebner, and T. H. Osborne, Phys. Rev. Lett. 112, 125002 (2014).

[38] F. Ryter, L. Barrera Orte, B. Kurzan, R. M. McDermott, G. Tardini, E. Viezzer, M. Bernert, R. Fischer, and The ASDEX Upgrade Team, Nucl. Fusion 54, 083003 (2014). 